\title{
Towards a consistent numerical compressible non-hydrostatic model using generalized Hamiltonian tools
}

\author{
Almut Gassmann ${ }^{\mathrm{a} *}$ and Hans-Joachim Herzog ${ }^{\mathrm{b}}$ \\ a Max Planck Institute for Meteorology, Hamburg, Germany \\ b Deutscher Wetterdienst, Potsdam, Germany
}

\begin{abstract}
A set of compressible non-hydrostatic equations for a turbulence-averaged model atmosphere comprising dry air and water in three phases plus precipitating fluxes is presented, in which common approximations are introduced in such a way that no inconsistencies occur in the associated budget equations for energy, mass and Ertel's potential vorticity. These conservation properties are a prerequisite for any climate simulation or NWP model.

It is shown that a Poisson bracket form for the ideal fluid part of the full-physics equation set can be found, while turbulent friction and diabatic heating are added as separate 'dissipative' terms. This Poisson bracket is represented as a sum of a two-fold antisymmetric triple bracket (a Nambu bracket represented as helicity bracket) plus two antisymmetric brackets (so-called mass and thermodynamic brackets of the Poisson type).

The advantage of this approach is that the given conservation properties and the structure of the brackets provide a good strategy for the construction of their discrete analogues. It is shown how discrete brackets are constructed to retain their antisymmetric properties throughout the spatial discretisation process, and a method is demonstrated how the time scheme can also be incorporated in this philosophy. Copyright (C) 2008 Royal Meteorological Society
\end{abstract}

KEY WORDS NWP; conservable quantity; Poisson bracket

Received 3 December 2007; Revised 23 June 2008; Accepted 26 June 2008

\section{Introduction}

When designing an atmospheric numerical model for the purpose of numerical weather prediction (NWP) and climate simulations, a careful formulation of the continuous model equations is obviously the first step. Closely connected, the construction of a physically adequate numerical scheme follows as the second step to form eventually a satisfactory discrete analogue. The paper deals with both of these main points. First, we start by formulating a continuous model equation set, which is consistent with respect to energy, mass, and Ertel's (1942) potential vorticity (EPV) conservation. It is written in Poisson bracket form applying elements from Névir's $(1998,2004)$ atmospheric energy-vorticity theory and also the Hamiltonian description from Morrison (1998) for an ideal fluid. Second, we are going to propose a method to construct the discrete analogue of the Poisson brackets both in space and time. This philosophy allows us to retain the continuous conservation properties as already demonstrated impressively by Salmon $(2004,2005,2007)$ concerning the spatial discretisation.

From the point of view of theoretical meteorology, the formulation of continuous model equations seems well established. Nevertheless, even today one becomes

* Correspondence to: Almut Gassmann, Max Planck Institute for Meteorology, Bundesstr. 53, 20146 Hamburg, Germany.

E-mail: Almut.Gassmann@zmaw.de aware that notorious problems emerge when trying to use in NWP in a consistent manner what is available from theory. In a recent paper, Thuburn (2008) has carefully reviewed this dilemma of how to design atmospheric models under the aspect of conservation properties.

In the present paper, we take up this problem for the compressible non-hydrostatic equations. This equation type became of common interest for modelling because of its suitability for atmospheric simulations over a wide range of meteorological phenomena from planetary down to local scales. Different non-hydrostatic regional models currently exist as research and operational weather forecasting models. Concerning global modelling, a notable development is taking place in Japan (Satoh 2002, 2003), emphasizing the careful inclusion of moist processes and conservative properties in the numerical scheme. Ultimately, this development is directed towards a global climate model with improved cloud-radiation interaction. A particular example of the application of compressible non-hydrostatic equations over the globe is the Unified Model of the UK Met Office (Davies et al., 2005) based on thorough research work with a fairly general conception to use it as a global NWP model, and for climate simulations as well. In our case we are motivated to deal with this model type in a running project also to develop a new global NWP and climate simulation model, called ICON (ICOsahedral Non-hydrostatic general circulation model) (www.icon.enes.org). 
In the following approach we take advantage of our experience with the compressible non-hydrostatic LokalModell (LM) (Doms and Schättler, 2002; Gassmann and Herzog, 2007), which runs as a limited-area model operationally in the German Weather Service. The basic equations of the LM are in principle of rather general validity so as to use them also as a basis to formulate a global model. Our demanding approach begins with an equation set considering an atmosphere consisting of dry air and water vapour as gaseous components, with the addition of water in liquid and solid form (cloud drops, cloud ice, precipitating drops and ice particles). The conceptual way to take into account a multi-component system is borrowed from Wacker et al. (2006). Additionally, the complete equation system is written by applying a massweighted turbulence averaging (known as Hesselberg averaging). With minute approximations, this equation set serves us as a reference model, having mass, energy and EPV conservation. This is outlined in section 2.

Further, we introduce common approximations to arrive at realistic averaged model equations available in a more meteorological form with temperature and pressure as model variables. Molecular fluxes are neglected compared to the corresponding turbulent fluxes. In such a way, the model equations are equations of averaged quantities, where the molecular dissipation of kinetic energy is omitted and should be replaced by turbulent dissipation as a remedy to obtain energy conserving model equations. The mass conservation is simply fulfilled and it is shown what mass control conditions are necessary to be considered for the partial mass budget equations of the multi-component system (Wacker et al., 2006). It is shown that the EPV is also a conservable quantity. On this way, we have found a full-physics model equation set appropriate to apply the Hamiltonian tool. This is the issue in section 3 .

We invoke this theory because to the best of our belief a Poisson bracket form with its specific antisymmetric property offers an interesting new way to find conservative numerical analogues (Salmon, 2004, 2005, 2007). Although hardly recognised up to now in the common literature, it is important to note that Névir and Blender (1993), and then Névir (1998), have shown years ago a way to formulate Nambu brackets for different classes of atmospheric equations. We will show in section 4 that it is possible to find a turbulence-averaged compressible non-hydrostatic model equation set in Poisson-Nambu bracket form including all water constituents, precipitation fluxes, and diabatic sources/sinks in such a way that, in the limit case for an ideal fluid, the bracket form for a rotating atmosphere is exactly recovered (Névir, 1998). The authors were thus able to demonstrate that the Hamiltonian theory for an ideal fluid is actually applicable beyond this physical limitation. The bracket approach provides a compact functional evolution equation from which it is easy to derive corresponding model equation sets in a well-structured form using different reasonable model variables.
Salmon $(2004,2005,2007)$ was inspired by the idea to retain the antisymmetry properties of dynamic brackets during model discretisation, in particular for the shallowwater equations, using the Poisson bracket formulation first, and then the more general Nambu bracket approach to construct conservative spatial schemes. Sommer (2007, personal communication) has taken up these ideas and was successful in finding a discret Nambu bracket form of a shallow-water model over the globe on the triangular/hexagonal ICON grid. It is interesting to keep in mind how the original and more intuitive Jacobian approach from Arakawa (1966) now becomes raised to the level of a much more general philosophy with very practical meaning for up-to-date model development. In this sense Dubinkina and Frank (2007) have reconsidered Arakawa's famous discretization using the Nambu bracket formalism for long-term integrations of the quasigeostrophic potential vorticity model with orographic forcing having conservation of discrete approximations of energy and/or enstrophy. In section 5 we show a method of formulating a numerical scheme both in space and time using the Poisson-Nambu bracket form of the consistently derived non-hydrostatic compressible equations. Thereby, we closely follow Salmon's suggestions representing global integrals by global sums, and considering the rule of integration by parts in the spatial numerics. But here, the rule of integration by parts in the time scheme is also required if one of the brackets occurs in different prognostic model equations. To confirm the suggested method, first results of one-dimensional experiments are shown in section 6 .

\section{Reference equation set for a heterogeneous system}

As a first point, we think of a quite general compressible non-hydrostatic equation set describing a atmospheric heterogeneous flow regime. Heterogeneity means here a two-component system consisting of dry air and water. Water is assumed to occur in all three phases including precipitating drops and ice particles. With reference to Wacker et al. (2006), partial densities $\varrho_{i}$ are introduced and summed up to total density for this atmospheric mixture, $\varrho=\Sigma \varrho_{i}$, where the subscripts $i=\mathrm{d}$, v, l, f, r, $\mathrm{p}$ refer to dry air, water vapour, liquid and frozen cloud particles, rain drops and precipitating ice particles (snow, graupel, etc.), respectively. A reference velocity vector is defined as a weighted mean, $\mathbf{v}=\Sigma \varrho_{i} \mathbf{v}_{i} / \varrho$. Due to this, an equation set for the mixture can be found, where the momentum equation, the continuity equation and the internal energy equation in this system are each formed as a sum from their separate component equations. We follow here the theoretical foundation of Wacker et al. (2006) who discuss the Cauchy form conservation of the momentum equation for a multicomponent system in their section 3 with further supporting references such as Gyarmati (1970) and Doms and Herbert (1985). Lange (2002) presents the same problem in his textbook. An alternative foundation is given by Bannon (2002) who 
considered a different reference velocity with respect to dry air, but we do not pursue that approach. In a further procedure, we carry out a turbulence (Reynolds) averaging for these equations, where a barycentric mean (Hesselberg, 1925) with respect to the total density is used, $\widehat{\psi}=\overline{\varrho \psi} / \bar{\varrho}$, having $\psi=\widehat{\psi}+\psi^{\prime \prime}$ for the considered variables. In this way we arrive at a sufficiently general equation set:

$$
\begin{aligned}
\bar{\varrho} \frac{\widehat{\mathrm{d}} \widehat{\mathbf{d}}}{\mathrm{d} t}=-\nabla \bar{p}-\bar{\varrho} \nabla \Phi-2 \boldsymbol{\Omega} \times \bar{\varrho} \widehat{\mathbf{v}} \\
\quad+\nabla \cdot\left(\overline{\boldsymbol{F}}-\overline{\varrho \mathbf{v}^{\prime \prime} \mathbf{v}^{\prime \prime}}\right), \\
\frac{\widehat{\mathrm{d}} \bar{\varrho}}{\mathrm{d} t}=-\bar{\varrho} \nabla \cdot \widehat{\mathbf{v}}, \\
\bar{\varrho} \frac{\widehat{\mathrm{d}} \widehat{u}}{\mathrm{~d} t}=-\bar{p} \nabla \cdot \widehat{\mathbf{v}}-\overline{p \nabla \cdot \mathbf{v}^{\prime \prime}}-\nabla \cdot\left(\overline{\mathbf{W}}+\overline{\varrho u^{\prime \prime} \mathbf{v}^{\prime \prime}}\right) \\
\quad+\overline{\boldsymbol{F}} \cdot \nabla \widehat{\mathbf{v}}+\overline{F \cdot \nabla \mathbf{v}^{\prime \prime}}, \\
\bar{\varrho} \frac{\widehat{\mathrm{d}} \widehat{q}_{i}}{\mathrm{~d} t}=-\nabla \cdot\left(\overline{\mathbf{J}_{i}}+\overline{\varrho q_{i}^{\prime \prime} \mathbf{v}^{\prime \prime}}\right)+\bar{Q}_{i} .
\end{aligned}
$$

In order to spare writing, the unaveraged multicomponent equations originally assumed are easily obtained by omitting the turbulence averaging symbols and all the turbulence flux terms in Equations (1)-(4). The turbulence-averaged equations are the momentum equation (1), the continuity equation (2), the budget equation for the mean internal (heat) energy, $\widehat{u},(3)$, and budget equations for the partial mass fractions, $\widehat{q_{i}}=\overline{\varrho_{i}} / \bar{\varrho}$, (4), having $\Sigma \widehat{q}_{i}=1$. It is important to note that, as a mass control condition, the sum of the $\widehat{q_{i}}$ budget equations over all $i$ have to yield the continuity equation (2), which implies $\Sigma \bar{Q}_{i}=0\left(\bar{Q}_{\mathrm{d}}=0\right), \Sigma \overline{\mathbf{J}}_{i}=0, \Sigma \overline{\varrho q_{i}^{\prime \prime} \mathbf{v}^{\prime \prime}}=0$.

\begin{tabular}{|c|c|}
\hline Symbol & Explanation \\
\hline $\mathbf{v}_{i}$ & velocity of $i$ th constituent \\
\hline $\mathbf{v}_{i}^{(d)}:=\mathbf{v}_{i}-\mathbf{v}$ & diffusion velocity of $i$ th constituent \\
\hline$\Omega$ & angular velocity of the Earth \\
\hline$\Phi$ & geopotential \\
\hline$Q_{i}$ & source/sink terms \\
\hline $\mathbf{J}_{i}=\varrho_{i} \mathbf{v}_{i}^{(d)}$ & diffusion flux of $i$ th constituent \\
\hline$\underline{F}$ & viscous friction tensor \\
\hline$\overline{\mathbf{J}_{u}}$ & heat diffusion flux vector \\
\hline $\mathbf{R}$ & radiation flux vector \\
\hline $\mathbf{W}=\mathbf{J}_{u}+\mathbf{R}$ & composed heat flux vector \\
\hline$\underline{\varrho \mathbf{v}^{\prime \prime} \mathbf{v}^{\prime \prime}}$ & turbulent momentum flux tensor \\
\hline$\underline{\underline{Q u^{\prime \prime} \mathbf{v}^{\prime \prime}}}$ & turbulent heat flux vector \\
\hline$\varrho q_{i}^{\prime \prime} \mathbf{v}^{\prime \prime}$ & $\begin{array}{l}\text { turbulent vector flux of } i \text { th partial } \\
\text { mass fraction }\end{array}$ \\
\hline$\frac{\widehat{\mathrm{d}}}{\mathrm{d} t}=\frac{\partial}{\partial t}+\widehat{\mathbf{v}} \cdot \nabla$ & $\begin{array}{l}\text { average individual time change } \\
\text { operator }\end{array}$ \\
\hline
\end{tabular}
Further explanations of symbols and terms in these equations are given in Table I. In Equation (3) and in subsequent equations, the notation .. means the double scalar product between tensors.

Table I. Explanation of symbols.
Such atmospheric model equations do not govern, but rather attempt to represent real processes in the atmosphere. Such forms will never be exact, and approximations are unavoidable. These approximations must not violate the most important conservation properties regarding mass, energy and EPV. This should be a guide, when we are going to introduce further simplifications towards more practical model equations compared to the reference equation set. Our model equations are to be valid for turbulence-averaged variables with reference to (1)-(4). This is an adequate assumption in view of realistic modelling accompanied by discretisations in space and time, where the turbulent flux terms represent subgrid-scale processes determined by parametrizations. We omit now as usual the viscous friction tensor against the turbulent momentum flux tensor, $-\overline{\varrho \mathbf{v}^{\prime \prime} \mathbf{v}^{\prime \prime}} \gg \overline{\boldsymbol{F}}$, and so the molecular heat flux against the turbulent heat flux, $\varrho u^{\prime \prime} \mathbf{v}^{\prime \prime} \gg \mathbf{J}_{u}$, from which we also have $\overline{\mathbf{W}} \Rightarrow \overline{\mathbf{R}}$. In the $\widehat{q}_{i}$ budget equations (4), however, the diffusion fluxes $\overline{\mathbf{J}}_{i}$ must not be dropped against the turbulent fluxes $\overline{\varrho q_{i}^{\prime \prime} \mathbf{v}^{\prime \prime}}$ in view of significant sedimentation (precipitation) fluxes. From energetics reasoning, it is acceptable to neglect in the heat energy equation (3) the direct energy transformation from mean kinetic energy to mean internal energy compared to the molecular dissipation term, $\overline{\boldsymbol{F} \cdot \nabla \mathbf{v}^{\prime \prime}} \gg \underline{\boldsymbol{F}} \cdot \nabla \widehat{\mathbf{v}}$.

With these approximations the averaged equation system becomes

$$
\begin{aligned}
\bar{\varrho} \frac{\widehat{\mathrm{d}} \widehat{\mathbf{v}}}{\mathrm{d} t}=-\nabla \bar{p}-\bar{\varrho} \nabla \Phi-2 \boldsymbol{\Omega} \times \bar{\varrho} \widehat{\mathbf{v}}+\nabla \cdot\left(-\overline{\varrho \mathbf{v}^{\prime \prime} \mathbf{v}^{\prime \prime}}\right), \\
\frac{\widehat{\mathrm{d}} \bar{\varrho}}{\mathrm{d} t}=-\bar{\varrho} \nabla \cdot \widehat{\mathbf{v}}, \\
\bar{\varrho} \frac{\widehat{\mathrm{d}} \widehat{u}}{\mathrm{~d} t}=-\nabla \cdot\left(\overline{\mathbf{R}}+\overline{\varrho u^{\prime \prime} \mathbf{v}^{\prime \prime}}\right)-\bar{p} \nabla \cdot \widehat{\mathbf{v}}-\overline{p \nabla \cdot \mathbf{v}^{\prime \prime}} \\
\quad+\underline{\boldsymbol{F} \cdot \nabla \mathbf{v}^{\prime \prime}}, \\
\widehat{\varrho} \frac{\mathrm{d}_{q_{i}}}{\mathrm{~d} t}=-\nabla \cdot\left(\overline{\mathbf{J}_{i}}+\overline{\varrho q_{i}^{\prime \prime} \mathbf{v}^{\prime \prime}}\right)+\bar{Q}_{i} .
\end{aligned}
$$

From the internal energy budget equation (7) and a mechanical energy budget equation immediately derived from the momentum equation (5),

$$
\begin{aligned}
\bar{\varrho} \frac{\widehat{\mathrm{d}}}{\mathrm{d} t}\left(\frac{\widehat{\mathbf{v}}^{2}}{2}+\Phi\right)=- & \nabla \cdot\left\{\bar{p} \widehat{\mathbf{v}}-\left(-\overline{\varrho \mathbf{v}^{\prime \prime} \mathbf{v}^{\prime \prime}}\right) \cdot \widehat{\mathbf{v}}\right\}+\bar{p} \nabla \cdot \widehat{\mathbf{v}} \\
& -\left(-\overline{\varrho \mathbf{v}^{\prime \prime} \mathbf{v}^{\prime \prime}}\right) \cdots \nabla \widehat{\mathbf{v}}
\end{aligned}
$$

a consistency requirement for the conservation of total energy budget as the sum of (7) and (9) can be inferred. Obviously, it reads

$$
\left(-\overline{\varrho \mathbf{v}^{\prime \prime} \mathbf{v}^{\prime \prime}}\right) \cdots \nabla \widehat{\mathbf{v}}+\overline{p \nabla \cdot \mathbf{v}^{\prime \prime}}-\overline{\boldsymbol{F} \cdot \nabla \mathbf{v}^{\prime \prime}}=0 .
$$

This requirement (10) can be interpreted as the equilibrium case of a rudimentary mean turbulent kinetic energy equation formed from three terms which are shear 
production, buoyancy production and molecular dissipation. With this reasoning we arrive eventually at an energy-consistent equation set, using the abbreviation $\varepsilon=-\overline{\varrho \mathbf{v}^{\prime \prime} \mathbf{v}^{\prime \prime}} \cdot \cdot \nabla \widehat{\mathbf{v}}>0$,

$$
\begin{aligned}
\bar{\varrho} \frac{\widehat{\mathrm{d}} \widehat{\mathrm{d}}}{\mathrm{d} t} & =-\nabla \bar{p}-\bar{\varrho} \nabla \Phi-2 \boldsymbol{\Omega} \times \widehat{\varrho} \widehat{\mathbf{v}}-\nabla \cdot \overline{\varrho \mathbf{v}^{\prime \prime} \mathbf{v}^{\prime \prime}}, \\
\frac{\widehat{\mathrm{d}} \bar{\varrho}}{\mathrm{d} t} & =-\bar{\varrho} \nabla \cdot \widehat{\mathbf{v}}, \\
\bar{\varrho} \frac{\mathrm{d} \widehat{u}}{\mathrm{~d} t} & =-\bar{p} \nabla \cdot \widehat{\mathbf{v}}-\nabla \cdot\left(\overline{\mathbf{R}}+\overline{\varrho u^{\prime \prime} \mathbf{v}^{\prime \prime}}\right)+\varepsilon, \\
\bar{\varrho} \frac{\widehat{\mathrm{d}} \widehat{q_{i}}}{\mathrm{~d} t} & =-\nabla \cdot\left(\overline{\mathbf{J}_{i}}+\overline{\varrho q_{i}^{\prime \prime} \mathbf{v}^{\prime \prime}}\right)+\overline{Q_{i}},
\end{aligned}
$$

together with a closed total energy budget

$$
\begin{aligned}
& \bar{\varrho} \frac{\widehat{\mathrm{d}} t}{}\left(\frac{\widehat{\mathbf{v}}^{2}}{2}+\Phi+\widehat{u}\right) \\
& \quad=-\nabla \cdot\left(\bar{p} \widehat{\mathbf{v}}+\overline{\varrho \mathbf{v}^{\prime \prime} \mathbf{v}^{\prime \prime}} \cdot \widehat{\mathbf{v}}+\overline{\mathbf{R}}+\overline{\varrho u^{\prime \prime} \mathbf{v}^{\prime \prime}}\right) .
\end{aligned}
$$

In addition to this, we formulate also the budget equation for the EPV from the present system using (11) and (12) with known operations. It follows that

$$
\begin{aligned}
& \bar{\varrho} \frac{\widehat{\mathrm{d}}}{\mathrm{d} t}\left(\frac{\widehat{\omega}_{\mathrm{a}} \cdot \nabla \widehat{\psi}}{\bar{\varrho}}\right)=-\nabla \cdot\left[\widehat{\psi}\left(\nabla \frac{1}{\bar{\varrho}} \times \nabla \bar{p}\right)\right. \\
& \left.-\widehat{\omega}_{\mathrm{a}} \frac{\widehat{\mathrm{d}} \widehat{\psi}}{\mathrm{d} t}-\widehat{\psi}\left(\nabla \times \mathbf{f}_{\mathrm{r}}\right)\right] \text {. }
\end{aligned}
$$

For the vector $\mathbf{f}_{\mathrm{r}}$ we have

$$
\mathbf{f}_{\mathrm{r}}=-\frac{\overline{\omega^{\prime \prime} \times \varrho \mathbf{v}^{\prime \prime}}}{\bar{\varrho}}-\frac{1}{\bar{\varrho}} \overline{\varrho \nabla \frac{\mathbf{v}^{\prime \prime 2}}{2}}=-\frac{1}{\varrho} \nabla \cdot \bar{\varrho} \overline{\mathbf{v}^{\prime \prime} \mathbf{v}^{\prime \prime}},
$$

and, furthermore, $\widehat{\psi}$ is an arbitrary scalar turbulenceaveraged function, $\widehat{\boldsymbol{\omega}}_{\mathrm{a}}=\widehat{\boldsymbol{\omega}}+2 \boldsymbol{\Omega}=\nabla \times \widehat{\mathbf{v}}+2 \boldsymbol{\Omega}$ is the mean absolute vorticity vector, and $\omega^{\prime \prime}=\nabla \times \mathbf{v}^{\prime \prime}$ is the turbulent vorticity vector. As can be seen, the EPV is also a conservative quantity. It connects the vorticity nature of the turbulent flow with the total mass conservation and the moist thermodynamics (replacing $\widehat{\psi}$ by a modelspecific thermodynamic quantity which is still left open here).

\section{Towards model equations applicable to a Poisson bracket form}

In the following we are interested in a more meteorological form of the equation set (11)-(14) aiming at the model variables $\widehat{\mathbf{v}}, \widehat{T}, \bar{p}, \widehat{q_{i}}$ instead of $\widehat{\mathbf{v}}, \bar{\varrho}, \widehat{u}, \widehat{q_{i}}$. For that purpose the turbulence-averaged specific enthalpy $\widehat{h}$ is introduced due to its relation to pressure and specific internal energy,

$$
\widehat{\varrho} \widehat{h}=\widehat{\varrho} \widehat{u}+\bar{p} .
$$

Here, we make use of the assumption that the equation of state and so the nonlinear relation for the total specific enthalpy (20) are valid for averaged quantities as an analogue to the unaveraged relations (cf. Herbert, 1975; Doms and Herbert, 1985)

$$
\bar{p}=R_{\mathrm{d}} \bar{\varrho} \widehat{T}(1+\widehat{\alpha}) .
$$

$\widehat{\alpha}$ means the averaged virtual increment,

$$
\widehat{\alpha}=\left(\frac{R_{\mathrm{v}}}{R_{\mathrm{d}}}-1\right) \widehat{q_{\mathrm{v}}}-\widehat{q_{\mathrm{l}}}-\widehat{q_{\mathrm{f}}}-\widehat{q_{\mathrm{r}}}-\widehat{q_{\mathrm{p}}} .
$$

In a similar manner we yield for the nonlinear relation of the total specific enthalpy of the assumed mixture

$$
\widehat{h}=\sum \widehat{h}_{i} \widehat{q}_{i},
$$

with

$$
\widehat{h_{i}}=h_{0 i}+c_{p i}\left(\widehat{T}-T_{0}\right)
$$

for the specific enthalpy of the $i$ th component, and for the specific heat capacities we have

$$
\widehat{c}_{p}=\sum c_{p i} \widehat{q}_{i} ; \quad \widehat{c}_{v}=\sum c_{v i} \widehat{q}_{i} .
$$

By use of (17)-(22), we arrive after a straightforward analysis from (13) and (12) at the desired prognostic equations for $\widehat{T}$ and $\bar{p}$. This so-called meteorological form of the consistent equation set reads

$$
\bar{\varrho} \frac{\widehat{\mathrm{d}} \widehat{\mathbf{v}}}{\mathrm{d} t}=-\nabla \bar{p}-\bar{\varrho} \nabla \Phi-2 \boldsymbol{\Omega} \times \widehat{\varrho} \widehat{\mathbf{v}}-\nabla \cdot \overline{\varrho \mathbf{v}^{\prime \prime} \mathbf{v}^{\prime \prime}},
$$

$$
\begin{aligned}
\widehat{c_{v}} \bar{\varrho} \frac{\widehat{\mathrm{d}} \widehat{T}}{\mathrm{~d} t} & =-\bar{p} \nabla \cdot \widehat{\mathbf{v}}+\bar{Q}_{h}+\bar{Q}_{m}, \\
\frac{\widehat{\mathrm{d}} \bar{p}}{\mathrm{~d} t} & =-\frac{\widehat{c_{p}}}{\widehat{c}_{v}} \bar{p} \nabla \cdot \widehat{\mathbf{v}}+\left(\frac{\widehat{c}_{p}}{\widehat{c}_{v}}-1\right) \bar{Q}_{h}+\frac{\widehat{c}_{p}}{\widehat{c}_{v}} \bar{Q}_{m}, \\
\bar{\varrho} \frac{\widehat{\mathrm{d}} \widehat{q_{i}}}{\mathrm{~d} t} & =-\nabla \cdot\left(\overline{\mathbf{J}_{i}}+\overline{\varrho q_{i}^{\prime \prime} \mathbf{v}^{\prime \prime}}\right)+\bar{Q}_{i}, \\
\bar{p} & =R_{\mathrm{d}} \bar{\varrho} \widehat{T}(1+\widehat{\alpha}) .
\end{aligned}
$$

The thermal source function $\bar{Q}_{h}$ and the moisture source function $\bar{Q}_{\mathrm{m}}$ are defined as

$$
\begin{aligned}
& \bar{Q}_{h}=-\nabla \cdot\left(\overline{\mathbf{R}}+\overline{\varrho u^{\prime \prime} \mathbf{v}^{\prime \prime}}\right)-\sum \widehat{h_{i}} \bar{\varrho} \frac{\widehat{\mathrm{d}} \widehat{q_{i}}}{\mathrm{~d} t}+\varepsilon, \\
& \bar{Q}_{\mathrm{m}}=R_{\mathrm{d}} \widehat{T} \bar{\varrho} \frac{\widehat{\mathrm{d}} \widehat{\alpha}}{\mathrm{d} t} .
\end{aligned}
$$

However, the derivation of the equation system (23)(29) needs some more attention concerning mass conservation when sedimentation fluxes are incorporated in the water budget equations (26). Invoking the approach of Wacker et al. (2006) (and also Catry et al., 2007), mass control conditions are necessary to be taken into 
account. One of them, $\Sigma \overline{\mathbf{J}_{i}}=0$, is here of particular interest. Assuming diffusion fluxes given only in the vertical direction, the precipitation fluxes (rain, snow, etc.) are to be described by a common ansatz from Rogers and Yau (1989), $\overline{S_{j}}=-\overline{\mathbf{J}_{j}}=\bar{\varrho} \widehat{q}_{j} \widehat{V}_{j}^{\mathrm{T}}$ for $j=\mathrm{r}$, p, where $\widehat{V}_{j}^{\mathrm{T}}$ is a terminal fall velocity to be parametrized. In order not to violate mass conservation, it is important to consider the rest of the diffusion fluxes, too, which have to play a compensating role. In the most simple way, these compensating fluxes may be read as $\overline{J_{i}}=\bar{\varrho} \widehat{q}_{i} \widehat{w}^{(d)}$ for $i=\mathrm{d}, \mathrm{v}, 1, \mathrm{f}$, assuming the same vertical diffusion velocity $\widehat{w}^{(\mathrm{d})}$ instead of different $\widehat{w}_{i}^{(\mathrm{d})}$. Thus we have

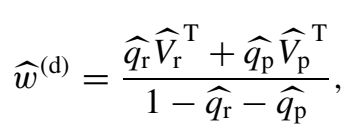

and the $\widehat{q_{i}}$ budget equations (26) read more specifically

$$
\begin{aligned}
\bar{\varrho} \frac{\widehat{\mathrm{d}} \widehat{q}_{i}}{\mathrm{~d} t} & =-\nabla \cdot \overline{\varrho q_{i}^{\prime \prime} \mathbf{v}^{\prime \prime}}-\frac{\partial \varrho \widehat{\varrho} \widehat{q_{i}} \widehat{w}^{(\mathrm{d})}}{\partial z}+\overline{Q_{i}} ; \\
i & =\mathrm{d}, \mathrm{v}, \mathrm{l}, \mathrm{f}, \\
\bar{\varrho} \frac{\widehat{\mathrm{d}} \widehat{q}_{j}}{\mathrm{~d} t} & =-\nabla \cdot \overline{\varrho q_{j}^{\prime \prime} \mathbf{v}^{\prime \prime}}+\frac{\partial \bar{\varrho} \widehat{q}_{j} \widehat{V}_{j}^{\mathrm{T}}}{\partial z}+\overline{Q_{j}} ; \\
j & =\mathrm{r}, \mathrm{p} .
\end{aligned}
$$

The other mass control conditions are $\Sigma \widehat{q_{i}}=1$, $\Sigma \overline{\varrho q_{i}^{\prime \prime} \mathbf{v}^{\prime \prime}}=0$, and $\Sigma \overline{Q_{i}}=0$ with $\overline{Q_{\mathrm{d}}}=0$.

In a further step the 'meteorological' system (23) (29) is now transformed into an obliging form in view of a Poisson bracket construction on the base of these equations. The works of Morrison (1998) and of Névir (1998) serve as an example for the following analysis, though both authors have treated an ideal fluid compared to our much more realistic equation set. The treatment of Névir is from the meteorological point of view more interesting, because he includes the Coriolis effects, while Morrison has discussed a non-rotating system. For the following, we switch to the Eulerian form of our equation set, and we take into account density $\bar{\varrho}$ and virtual potential temperature $\widehat{\theta_{v}}$ as prognostic variables instead of $\widehat{T}$ and $\bar{p}$ in the previous set. The reason why we have chosen $\widehat{\theta_{v}}$ is to arrive in (31) at a tractable form of the pressure gradient term, and so to obtain simple functional derivatives in (41) for the envisaged construction of a Poisson bracket form.

An important point is here the formulation of the momentum equation, where the advection term is identically reformulated due to the so-called Lamb transformation,

$$
\mathbf{v} \cdot \nabla \mathbf{v}=\omega \times \mathbf{v}+\nabla\left(\frac{1}{2} \mathbf{v}^{2}\right)
$$

We have decided to split off the advection term into a rotational term plus gradient of kinetic energy in order to unveil the ubiquitous vorticity process due to the rotational term, which otherwise would remain hidden. It is convenient to incorporate the Coriolis terms into one rotational term. In this point we follow the philosophy of Névir (1998). Moreover, the equation set is to be formulated in such a manner that in the limit case the equations for an ideal fluid are exactly recovered. The equation system equivalent to (23)-(29) may be written in the following form

$$
\begin{aligned}
\frac{\partial \widehat{\mathbf{v}}}{\partial t} & =-\widehat{\boldsymbol{\omega}}_{\mathrm{a}} \times \widehat{\mathbf{v}}-\nabla\left(\frac{1}{2} \widehat{\mathbf{v}}^{2}+\Phi\right)-\widehat{\theta}_{v} \nabla\left(c_{p \mathrm{~d}} \bar{\pi}\right)+\mathbf{f}_{\mathrm{r}} \\
\frac{\partial \bar{\varrho}}{\partial t} & =-\nabla \cdot(\widehat{\varrho} \widehat{\mathbf{v}}) \\
\frac{\partial\left(\bar{\varrho} \widehat{\theta_{v}}\right)}{\partial t} & =-\nabla \cdot\left(\widehat{\theta_{v}} \widehat{\varrho} \widehat{\mathbf{v}}\right)+\bar{\varrho} Q^{\left(\theta_{v}\right)} \\
\frac{\partial\left(\widehat{\varrho} \widehat{q}_{i}\right.}{\partial t} & =-\nabla \cdot\left(\bar{\varrho} \widehat{q_{i}} \widehat{\mathbf{v}}+\overline{\mathbf{J}}_{i}+\overline{\varrho q_{i}^{\prime \prime} \mathbf{v}^{\prime \prime}}\right)+\bar{Q}_{i} \\
\bar{p} & =R_{\mathrm{d}} \bar{\varrho} \widehat{T_{v}}, \\
\widehat{\theta_{v}} & =\widehat{T}_{v}\left(\frac{p_{00}}{\bar{p}}\right)^{\frac{R_{\mathrm{d}}}{c_{p \mathrm{~d}}}}=\frac{\widehat{T}_{v}}{\bar{\pi}}
\end{aligned}
$$

$\bar{\pi}$ is the Exner function as usual. The source function $Q^{\left(\theta_{v}\right)}$ in the $\widehat{\theta}_{v}$ equation (33) is defined by

$$
\begin{aligned}
c_{p \mathrm{~d}} \bar{\pi} \bar{\varrho} Q^{\left(\theta_{v}\right)}=( & \left.+\frac{c_{p \mathrm{~d}}(1+\widehat{\alpha})-\widehat{c}_{p}}{\widehat{c}_{v}}\right) \bar{Q}_{h} \\
& +\left(\frac{c_{p \mathrm{~d}}}{R_{\mathrm{d}}}+\frac{c_{p \mathrm{~d}}(1+\widehat{\alpha})-\widehat{c}_{p}}{\widehat{c}_{v}}\right) \bar{Q}_{\mathrm{m}} \\
& +\left(\frac{c_{p \mathrm{~d}}(1+\widehat{\alpha})-\widehat{c}_{p}}{\widehat{c}_{v}}\right)(-\bar{p} \nabla \cdot \widehat{\mathbf{v}}) .
\end{aligned}
$$

Strictly speaking, $Q^{\left(\theta_{v}\right)}$ is not a pure diabatic source function, because the third term on the RHS is actually a moist adiabatic term. Bannon (2002) found a similar form (his equation (7.5)).

From the equations (31)-(33), the associated EPV budget equation may now be derived. With reference to the more general form (15) we specify here $\widehat{\psi}=\widehat{\theta}_{v}$. This is the most suitable choice as discussed by Schubert et al. (2001). We obtain

$$
\frac{\partial}{\partial t}\left(\bar{\varrho} \Pi_{\mathrm{a}}\right)=-\nabla \cdot\left[\widehat{\varrho} \Pi_{\mathrm{a}} \widehat{\mathbf{v}}-\widehat{\boldsymbol{\omega}}_{\mathrm{a}} Q^{\left(\theta_{v}\right)}-\widehat{\theta}_{v}\left(\nabla \times \mathbf{f}_{\mathrm{r}}\right)\right],
$$

where the EPV is defined by

$$
\Pi_{\mathrm{a}}=\frac{1}{\bar{\varrho}}\left(\widehat{\boldsymbol{\omega}}_{\mathrm{a}} \cdot \nabla \widehat{\theta}_{v}\right)
$$

\section{Poisson bracket description}

Next we show how a Poisson bracket form can be found from the equation set (31)-(37). The Poisson formulation is here meant in a more limited sense. It 
concerns deliberately only those parts of the given fullphysics equation set which correspond in the limit case to an ideal fluid. The turbulent friction terms in the momentum equation and the heat and moisture source terms will be left away from such a bracket form. They are considered as additional 'dissipative' forcing terms added to the 'ideal-fluid' part. Making the notation simple enough, from now on the averaging symbols over all model variables will be dropped. We differ here from Névir (1998) in employing density times virtual potential temperature, $\widetilde{\theta_{v}}=\varrho \theta_{v}$, as a pressurelike variable instead of an entropy-like variable. We are going through the well-known Hamiltonian formulation. Here we quote a contribution from Bannon (2003), who has described a Hamiltonian form of an idealised binary geophysical fluid. Compared to him we introduce a more simplified Hamilton functional $\mathcal{H}$, which is not the complete Hamiltonian of the given system, but is it in the dry limit case with $T_{v} \rightarrow T$. It reads

$$
\mathcal{H}[\mathbf{u}]=\int_{V}\left(\frac{1}{2} \varrho \mathbf{v}^{2}+\varrho \Phi+\varrho c_{v \mathrm{~d}} T_{v}\right) \mathrm{d} \tau,
$$

where we have defined the vector $\mathbf{u}=\left(\mathbf{v}, \varrho, \widetilde{\theta_{v}}\right)$. The functional derivations of $\mathcal{H}$ with respect to the variables $\mathbf{v}, \varrho, \widetilde{\theta_{v}}$ are then

$$
\frac{\delta \mathcal{H}}{\delta \mathbf{v}}=\varrho \mathbf{v}, \quad \frac{\delta \mathcal{H}}{\delta \varrho}=\frac{1}{2} \mathbf{v}^{2}+\Phi, \quad \frac{\delta \mathcal{H}}{\delta \widetilde{\theta}_{v}}=c_{p \mathrm{~d}} \pi,
$$

to form the Hamiltonian form of the system (31)-(37)

$$
\begin{aligned}
\frac{\partial \mathbf{v}}{\partial t} & =-\frac{\boldsymbol{\omega}_{\mathrm{a}}}{\varrho} \times \frac{\delta \mathcal{H}}{\delta \mathbf{v}}-\nabla \frac{\delta \mathcal{H}}{\delta \varrho}-\theta_{v} \nabla \frac{\delta \mathcal{H}}{\delta \widetilde{\theta}_{v}}+\mathbf{f}_{\mathrm{r}}, \\
\frac{\partial \varrho}{\partial t} & =-\nabla \cdot \frac{\delta \mathcal{H}}{\delta \mathbf{v}} \\
\frac{\partial \widetilde{\theta_{v}}}{\partial t} & =-\nabla \cdot\left(\theta_{v} \frac{\delta \mathcal{H}}{\delta \mathbf{v}}\right)+\varrho Q^{\left(\theta_{v}\right)}
\end{aligned}
$$

Here, the 'physical' terms $\mathbf{f}_{\mathrm{r}}$ and $Q^{\left(\theta_{v}\right)}$ are assumed to be prescribed, and we note that the budget equations for the water constituents $q_{i}$ are really not lost in this subsystem, because their summed effect is implied in the continuity equation for total density.

We can obviously rewrite the given system to obtain in compact form a general evolution equation for a functional $\mathcal{F}[\mathbf{u}]$. Thus we have

$$
\frac{\partial \mathcal{F}[\mathbf{u}]}{\partial t}=\{\mathcal{F}, \mathcal{H}\}+(\mathcal{F}, \mathbf{r})+\left(\mathcal{F}, Q^{\left(\theta_{v}\right)}\right) .
$$

Here, the noncanonical Poisson bracket reads

$$
\begin{aligned}
\{\mathcal{F}, \mathcal{H}\}=- & \int_{V} \frac{\delta \mathcal{F}}{\delta \mathbf{v}} \cdot\left(\frac{\omega_{\mathrm{a}}}{\varrho} \times \frac{\delta \mathcal{H}}{\delta \mathbf{v}}\right) \mathrm{d} \tau \\
& -\int_{V}\left[\frac{\delta \mathcal{F}}{\delta \varrho} \nabla \cdot \frac{\delta \mathcal{H}}{\delta \mathbf{v}}-\frac{\delta \mathcal{H}}{\delta \varrho} \nabla \cdot \frac{\delta \mathcal{F}}{\delta \mathbf{v}}\right] \mathrm{d} \tau \\
& -\int_{V}\left[\frac{\delta \mathcal{F}}{\delta \widetilde{\theta_{v}}} \nabla \cdot\left(\theta_{v} \frac{\delta \mathcal{H}}{\delta \mathbf{v}}\right)-\frac{\delta \mathcal{H}}{\delta \widetilde{\theta_{v}}} \nabla \cdot\left(\theta_{v} \frac{\delta \mathcal{F}}{\delta \mathbf{v}}\right)\right] \mathrm{d} \tau
\end{aligned}
$$

and in the present case real-fluid 'physical' brackets are added in (45) due to turbulent frictional and diverse moist and diabatic processes involved in $\mathbf{f}_{\mathrm{r}}$ and $Q^{\left(\theta_{v}\right)}$ :

$$
\begin{aligned}
\left(\mathcal{F}, \mathbf{f}_{\mathrm{r}}\right) & =\int_{V} \frac{\delta \mathcal{F}}{\delta \mathbf{v}} \cdot \mathbf{f}_{\mathrm{r}} \mathrm{d} \tau, \\
\left(\mathcal{F}, Q^{\left(\theta_{v}\right)}\right) & =\int_{V} \frac{\delta \mathcal{F}}{\delta \widetilde{\theta}_{v}} \varrho Q^{\left(\theta_{v}\right)} \mathrm{d} \tau .
\end{aligned}
$$

The upgrading process to come from (42)-(44) to (45) may be made more transparent by a formal transition $\delta \mathcal{F} / \delta \mathbf{u} \Leftrightarrow \delta\left(\mathbf{r}-\mathbf{r}^{\prime}\right)$ with $\mathcal{F} \Leftrightarrow \mathbf{u}$, and by use of the generalised chain rule of differentiation,

$$
\frac{\partial \mathcal{F}[\mathbf{u}]}{\partial t}=\int_{V} \frac{\delta \mathcal{F}}{\delta \mathbf{u}} \cdot \frac{\partial \mathbf{u}}{\partial t} \mathrm{~d} \tau .
$$

By interchanging $\mathcal{F}$ and $\mathcal{H}$ the antisymmetric property of the Poisson bracket is obvious, $\{\mathcal{F}, \mathcal{H}\}=-\{\mathcal{H}, \mathcal{F}\}$. As discussed by Morrison (1998, p.490), the direct relation between the Poisson bracket (46) and the equations (42)-(44) is hidden and becomes only obvious after performing some integrations by parts, and associated boundary terms must vanish (see also Bannon, 2003). Concerning the latter, we have assumed

$\int_{V} \nabla \cdot\left(\frac{\delta \mathcal{F}}{\delta \mathbf{v}} \frac{\delta \mathcal{H}}{\delta \varrho}\right) \mathrm{d} \tau=0, \quad \int_{V} \nabla \cdot\left(\theta_{v} \frac{\delta \mathcal{F}}{\delta \mathbf{v}} \frac{\delta \mathcal{H}}{\delta \widetilde{\theta}_{v}}\right) \mathrm{d} \tau=0$,

or the alternative form with interchanging $\mathcal{F}$ and $\mathcal{H}$. For a further discussion of the bracket form (46), four functionals (a mass functional $\mathcal{M}$, a theta functional $\Theta_{v}$, a helicity functional $h_{\mathrm{a}}$ and a EPV-functional $\mathcal{P}_{\mathrm{a}}$ ) are introduced. They are

$$
\begin{aligned}
\mathcal{M}=\int_{V} \varrho \mathrm{d} \tau, & \Theta_{v}=\int_{V} \tilde{\theta_{v}} \mathrm{~d} \tau, \\
h_{\mathrm{a}}=\frac{1}{2} \int_{V} \boldsymbol{\omega}_{\mathrm{a}} \cdot \mathbf{v}_{\mathrm{a}} \mathrm{d} \tau, & \mathcal{P}_{\mathrm{a}}=\int_{V} \varrho \Pi_{\mathrm{a}} \mathrm{d} \tau,
\end{aligned}
$$

and the functional derivatives relevant for operations further below are

$$
\frac{\delta \mathcal{M}}{\delta \varrho}=1, \frac{\delta \Theta_{v}}{\delta \widetilde{\theta}_{v}}=1, \frac{\delta h_{\mathrm{a}}}{\delta \mathbf{v}}=\omega_{\mathrm{a}} .
$$

In (52) we have $\mathbf{v}_{\mathrm{a}}=\mathbf{v}+\boldsymbol{\Omega} \times \mathbf{r}$, where $\mathbf{r}$ is a position vector. The first two derivatives in (53) are trivial, but the third is not. Névir (1998, and personal communication) introduced the helicity functional in (52) and has proven its functional derivation in (53). Following Névir, the first of the three integral terms in the Poisson bracket (46) can be rewritten by use of the helicity, which leads to a Nambu bracket,

$$
\left\{\mathcal{F}, h_{\mathrm{a}}, \mathcal{H}\right\}=-\int_{V} \frac{\delta \mathcal{F}}{\delta \mathbf{v}} \cdot\left(\frac{1}{\varrho} \frac{\delta h_{\mathrm{a}}}{\delta \mathbf{v}} \times \frac{\delta \mathcal{H}}{\delta \mathbf{v}}\right) \mathrm{d} \tau .
$$

The permutation of two quantities of $\mathcal{F}, h_{\mathrm{a}}$ and $\mathcal{H}$ shows the two-fold antisymmetry of this bracket which 
is the well-known property of a scalar triple product we meet here. Thus, we have

$$
\left\{\mathcal{F}, h_{\mathrm{a}}, \mathcal{H}\right\}=-\left\{h_{\mathrm{a}}, \mathcal{F}, \mathcal{H}\right\}=-\left\{\mathcal{F}, \mathcal{H}, h_{\mathrm{a}}\right\} .
$$

The two other integral terms are here defined as mass bracket and theta-bracket, which are simply antisymmetric:

$$
\begin{aligned}
\{\mathcal{F}, \mathcal{H}\}_{\varrho} & =-\int_{V}\left[\frac{\delta \mathcal{F}}{\delta \varrho} \nabla \cdot \frac{\delta \mathcal{H}}{\delta \mathbf{v}}-\frac{\delta \mathcal{H}}{\delta \varrho} \nabla \cdot \frac{\delta \mathcal{F}}{\delta \mathbf{v}}\right] \mathrm{d} \tau \\
& =-\int_{V}\left[\frac{\delta \mathcal{F}}{\delta \mathbf{v}} \cdot \nabla \frac{\delta \mathcal{H}}{\delta \varrho}-\frac{\delta \mathcal{H}}{\delta \mathbf{v}} \cdot \nabla \frac{\delta \mathcal{F}}{\delta \varrho}\right] \mathrm{d} \tau \\
\{\mathcal{F}, \mathcal{H}\}_{\widetilde{\theta}_{v}} & =-\int_{V}\left[\frac{\delta \mathcal{F}}{\delta \widetilde{\theta_{v}}} \nabla \cdot\left(\theta_{v} \frac{\delta \mathcal{H}}{\delta \mathbf{v}}\right)-\frac{\delta \mathcal{H}}{\delta \widetilde{\theta_{v}}} \nabla \cdot\left(\theta_{v} \frac{\delta \mathcal{F}}{\delta \mathbf{v}}\right)\right] \mathrm{d} \tau \\
& =-\int_{V} \theta_{v}\left[\frac{\delta \mathcal{F}}{\delta \mathbf{v}} \cdot \nabla \frac{\delta \mathcal{H}}{\delta \widetilde{\theta_{v}}}-\frac{\delta \mathcal{H}}{\delta \mathbf{v}} \cdot \nabla \frac{\delta \mathcal{F}}{\delta \widetilde{\theta_{v}}}\right] \mathrm{d} \tau
\end{aligned}
$$

As can be seen, for each of these two brackets an alternative form is possible, which is a 'divergence' form or a 'gradient' form, already valid in (46). This duality of gradient and divergence rests on the assumption of vanishing boundary values due to the assumption of $\int_{V} \nabla \cdot(\ldots) \mathrm{d} \tau=0$ for arguments under the divergence operator as already discussed above.

Thus, we consider the Poisson bracket as the sum of a Nambu bracket (helicity bracket) plus a mass bracket and a theta bracket with reference to (46), (54), (56) and (57),

$$
\{\mathcal{F}, \mathcal{H}\}=\left\{\mathcal{F}, h_{\mathrm{a}}, \mathcal{H}\right\}+\{\mathcal{F}, \mathcal{H}\}_{\varrho}+\{\mathcal{F}, \mathcal{H}\}_{\widetilde{\theta}_{v}} .
$$

Due to (58) the evolution equation (45) is considered in the form

$$
\begin{gathered}
\frac{\partial \mathcal{F}[\mathbf{u}]}{\partial t}=\left\{\mathcal{F}, h_{\mathrm{a}}, \mathcal{H}\right\}+\{\mathcal{F}, \mathcal{H}\}_{\varrho}+\{\mathcal{F}, \mathcal{H}\}_{\widetilde{\theta_{v}}} \\
+\left(\mathcal{F}, \mathbf{f}_{\mathbf{r}}\right)+\left(\mathcal{F}, Q^{\left(\theta_{v}\right)}\right) .
\end{gathered}
$$

It describes in compact form our full-physics model system. Though mass, energy and EPV conservation has already been shown, the bracket form (59) confirms these conservation properties in an elegant way once again. These brackets can easily be evaluated by substituting $\mathcal{H}$, $\mathcal{M}, \Theta_{v}, h_{\mathrm{a}}, \Pi_{\mathrm{a}}$ for the general functional $\mathcal{F}$. The upper part of Table II shows the result for each bracket. From the evolution equation (59) then follows:

$$
\begin{aligned}
& \frac{\partial \mathcal{H}}{\partial t}-\left(\mathcal{H}, \mathbf{f}_{\mathrm{r}}\right)-\left(\mathcal{H}, Q^{\left(\theta_{v}\right)}\right)=\frac{\partial \mathcal{H}^{*}}{\partial t}=0 . \\
& \frac{\partial \mathcal{M}}{\partial t}=0, \\
& \frac{\partial \Theta_{v}}{\partial t}=\int_{V} \varrho Q^{\left(\theta_{v}\right)} \mathrm{d} \tau, \\
& \frac{\partial h_{a}}{\partial t}=\int_{V} c_{p \mathrm{~d}} \pi \varrho \Pi_{\mathrm{a}} \mathrm{d} \tau+\int_{V} \omega_{\mathrm{a}} \cdot \mathbf{f}_{\mathrm{r}} \mathrm{d} \tau, \\
& \frac{\partial \Pi_{\mathrm{a}}}{\partial t}=0 .
\end{aligned}
$$

The relation (60) shows the energy conservation. It indicates that $\mathcal{H}$ is not the Hamilton functional of our full-physics system, but

$$
\mathcal{H}^{*}=\int_{V}\left(\frac{1}{2} \varrho \mathbf{v}^{2}+\varrho \Phi+\varrho u\right) \mathrm{d} \tau .
$$

\begin{tabular}{|c|c|c|c|c|c|}
\hline $\mathcal{F}$ & $\left\{\mathcal{F}, h_{\mathrm{a}}, \mathcal{H}\right\}$ & $\{\mathcal{F}, \mathcal{H}\}_{\varrho}$ & $\{\mathcal{F}, \mathcal{H}\}_{\widetilde{\theta}_{v}}$ & $\left(\mathcal{F}, \mathbf{f}_{\mathrm{r}}\right)$ & $\left(\mathcal{F}, Q^{\left(\theta_{v}\right)}\right)$ \\
\hline $\mathcal{H}$ & 0 & 0 & 0 & $\int_{V} \varrho \mathbf{v} \cdot \mathbf{f}_{\mathrm{r}} \mathrm{d} \tau$ & $\int_{V} c_{p \mathrm{~d}} \pi \varrho Q^{\left(\theta_{v}\right)} \mathrm{d} \tau$ \\
\hline $\mathcal{M}$ & 0 & 0 & 0 & 0 & 0 \\
\hline$\Theta_{v}$ & 0 & 0 & 0 & 0 & $\int_{V} \varrho Q^{\left(\theta_{v}\right)} \mathrm{d} \tau$ \\
\hline$h_{\mathrm{a}}$ & 0 & 0 & $\int_{V} c_{p \mathrm{~d}} \pi \varrho \Pi_{\mathrm{a}} \mathrm{d} \tau$ & $\int_{V} \omega_{\mathrm{a}} \cdot \mathbf{f}_{\mathrm{r}} \mathrm{d} \tau$ & 0 \\
\hline$\Pi_{\mathrm{a}}$ & 0 & 0 & 0 & 0 & 0 \\
\hline $\mathbf{v}$ & $-\omega_{\mathrm{a}} \times \mathbf{v}$ & $-\nabla\left(\frac{1}{2} \mathbf{v}^{2}+\Phi\right)$ & $-\theta_{v} \nabla\left(c_{p \mathrm{~d}} \pi\right)$ & $\mathbf{f}_{\mathrm{r}}$ & 0 \\
\hline$\varrho$ & 0 & $-\nabla^{2} \cdot(\varrho \mathbf{v})$ & 0 & 0 & 0 \\
\hline$\widetilde{\theta}_{v}$ & 0 & 0 & $-\nabla \cdot\left(\theta_{v} \varrho \mathbf{v}\right)$ & 0 & $\varrho Q^{\left(\theta_{v}\right)}$ \\
\hline$\pi$ & 0 & 0 & $-\frac{R_{\mathrm{d}}}{c_{v \mathrm{~d}}} \frac{\pi}{\theta_{v}} \nabla \cdot\left(\theta_{v} \varrho \mathbf{v}\right)$ & 0 & $\frac{R_{d}}{c_{v} \mathrm{~d}} \tilde{\theta}_{\theta} \varrho Q^{\left(\theta_{v}\right)}$ \\
\hline$p$ & 0 & 0 & $-\frac{c_{p \mathrm{~d}}}{c_{v \mathrm{~d}}} \frac{p}{\ddot{\theta}_{v}} \nabla \cdot\left(\theta_{v} \varrho \mathbf{v}\right)$ & 0 & $\frac{c_{p \mathrm{~d}}}{c_{v \mathrm{~d}}} \frac{p}{\tilde{\theta}^{v}} \varrho Q^{\left(\theta_{v}\right)}$ \\
\hline$\theta_{v}$ & 0 & $\frac{\theta_{v}}{\varrho} \nabla \cdot(\varrho \mathbf{v})$ & $-\frac{\theta_{v}}{\tilde{\theta}_{v}} \nabla \cdot\left(\theta_{v} \varrho \mathbf{v}\right)$ & 0 & $Q^{\left(\theta_{v}\right)}$ \\
\hline$T_{v}$ & 0 & $\frac{T_{v}}{\varrho} \nabla \cdot(\varrho \mathbf{v})$ & $-\frac{c_{p \mathrm{~d}}^{\sigma_{v}}}{c_{v \mathrm{~d}}} \frac{T_{v}}{\theta_{v}} \nabla \cdot\left(\theta_{v} \varrho \mathbf{v}\right)$ & 0 & $\frac{c_{p \mathrm{~d}}}{c_{v \mathrm{~d}}} \frac{T_{v}}{\theta_{v}} \varrho Q^{\left(\theta_{v}\right)}$ \\
\hline
\end{tabular}

However, from a practical point of view, it remains for us to use the bracket form expressed by $\mathcal{H}$ for our model development. The mass conservation expressed by (61) is a trivial result, and so the functional $\mathcal{P}_{\mathrm{a}}$ is according to (64) a conservable quantity which can also be inferred from the given brackets. According to (62), $\Theta_{v}$ is not conservative due to the global sum of the general source $Q^{\left(\theta_{v}\right)}$. We would refer to the fluid to be an ideal fluid, if these $Q^{\left(\theta_{v}\right)}$ contributions vanish everywhere. Although the absolute helicity $h_{\mathrm{a}}$ is an essential constituent in the Nambu bracket (54), it is in general not a conservable

Table II. Bracket evaluation according to (59) for different functionals $\mathcal{F}$. 
quantity. As the relation (63) indicates, the EPV and the turbulent friction are sources of helicity. Only for the special case of vanishing EPV in a frictionless flow regime is the helicity conservative, but otherwise not. The budget relation (63) follows easily from the upper bracket form of our model equation set, but was originally found and discussed by Kurgansky (2006).

The reason why we get through the Poisson bracket description is that the formulation of model equations becomes well-structured. The given brackets, namely the helicity bracket, the mass bracket, and the theta bracket, determine the structural position and the role of each term due to its association with one of these three brackets. This then opens a conception of how to construct a numerical scheme. In this context we start generating diverse model equations from the general form (59) by setting $\mathcal{F}$ as a functional of an appropriate model variable according to (49). This is contained in the second part of Table II. In all three cases of temperature variables, $\mathcal{F}=\left(\theta_{v} ; T_{v} ; \widetilde{\theta_{v}}=\varrho \theta_{v}\right)$, we observe that the helicity bracket and the turbulent frictional bracket do not occur. In the case $\mathcal{F}=\widetilde{\theta}_{v}$, the mass bracket is absent, too. The latter indicates that the variable $\widetilde{\theta}_{v}=\varrho \theta_{v}$ is a masked pressure variable $\left(\varrho \theta_{v} \sim p^{c_{\mathrm{vd}} / c_{p \mathrm{~d}}}\right)$. Thus, the result is similar to the pressure variables $p$ and $\pi$.

One can speculate from Table II how to make a reasonable composite of model equations to form a full equation set. The momentum equation is covered by all three brackets (helicity, mass, and theta bracket). The continuity equation should be involved, which is constituted solely by the mass bracket. The system should then be completed by a pressure-like equation choosing the prognostic variables $\theta_{v}=\varrho \theta_{v}, p$, or $\pi$. Each of these equations is represented by the theta bracket and not also by the mass bracket, which is already governing the continuity equation. This allows us to describe the equation set by a minimal number of brackets, while in case of a $T_{v}$ or $\theta_{v}$ equation the mass bracket also participates, which might be redundant concerning the computational effort.

The following discretisation process on the basis of the antisymmetric properties of these brackets as a guideline must not approximate the same bracket type differently in the various equations. The structure of the mass and theta brackets suggests that their discretisation is concentrated on the discretisation of the divergence and gradient operators which must be treated consistently as dual operators. The discretisation of the helicity bracket will be a particularly intricate matter with its scalar-triple-product structure and its specific property not producing kinetic energy but anisotropically redistributing it. Seen as a working hypothesis, the explicit representation of this bracket is a concession to the ubiquitous vorticity nature in the atmosphere, which might help to better simulate smaller-scale structures like convective structures, etc. For each of the three brackets, in view of the discretisation process we are confronted with their inherently nonlinear and three-dimensional structure.

\section{Concept for the numerical discretisation of the Poisson and Nambu brackets}

\subsection{Preliminaries to bracket discretisations}

In the previous sections we dealt with the careful formulation of the continuous model equations conserving mass, energy and EPV. The particular point of view was to find out a bracket form of those model equations, which provides a new approach to construct the discrete analogue of the model equations, taking advantage of the inherent structural properties of Poisson and Nambu brackets. This follows as the second part of our treatment, belonging seamlessly to our earlier analysis. Here, we concentrate on demonstrating methods rather than showing many details and final results.

Salmon $(2005,2007)$ was the first to propose a general method to construct discretised Nambu brackets. Before adopting this method for our three-dimensional flow equations, we have to specify the circumstances in which we want to apply it. Every discretisation requires us to specify some properties in advance. These are the grid, some basis functions - if working with Galerkin methods - and some basic operators. Nambu bracket discretisations are applicable within a variety of approaches concerning these fundamental decisions. Salmon and Talley (1989) have already pointed out that their ansatz for the Jacobian, which is actually a discretised Nambu bracket, 'applies to finite difference, finite elements, spectral truncations, or to any other general method of producing discrete approximations'. Since we have already a certain model application in mind, namely the ICON model, we restrict our investigations to the Arakawa-C/Lorenz grid staggering, which is widely used in NWP models. Even though the ICON model will use a triangular mesh and the derivations will be given with a wide generality, we will discuss the results by way of an example employing a more common quadrilateral mesh.

We now closely follow the approach given by Salmon and Talley (1989), and by Salmon (2004, 2005, 2007), who replaced the functional integrals and the Nambu bracket integrals by sums over grid boxes. The discrete form of the chain rule of differentiation (49) then yields

$$
\frac{\partial \mathcal{F}[\mathbf{u}]}{\partial t} \Rightarrow \sum_{m=1}^{M}\left(\frac{\delta \mathcal{F}}{\delta \mathbf{u}} \cdot \frac{\partial \mathbf{u}}{\partial t}\right)_{m} V_{m} .
$$

The discrete analogue of the functional derivative $\delta \mathcal{F} / \delta \mathbf{u}$ just selects individual grid points with a factor $1 / V_{m}$ ( $V_{m}$ is the $m$ th grid-box volume) and vanishes otherwise. With that approach, our method turns out to be similar to finite difference methods.

\subsection{Discrete operators}

First, we have to specify the spatial operators on the grid. We can interpret the grid as an arrangement of two grid types, a primal and a dual grid (Bonaventura 

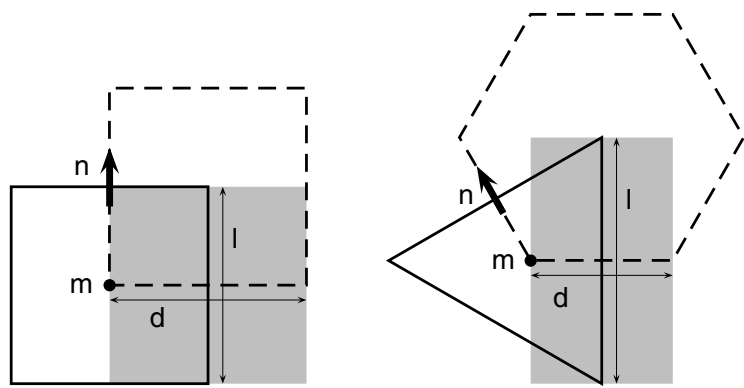

Figure 1. Pairs of primal (solid line borders) and dual (dot-dashed line borders) grids for quadrilateral and triangular primal meshes horizontal projection of grid cell volumes. Further explanations are given in the text.

and Ringler, 2005), which is shown in Figure 1 for a pair of quadrilateral meshes and a pair of triangular and hexagonal grids. For the ICON model, the triangular grid is referred to as the primal grid, but the opposite choice is also possible (Ničković et al., 2002; Torsvik et al., 2005). Each edge of a primal cell is orthogonal to one edge of a dual cell. We use here a horizontal C-grid arrangement, for which the normal wind component locations $n$ are defined at the centres of the primal grid edges (faces in three dimensions) and point normal to them. Their direction is thought of as pointing outwards with respect to the primal cell centre $m$. The scalar quantities are to be found at the cell centres $m$. In three dimensions, we combine a horizontal C-grid with a vertical L-grid. Then, the vorticity components are placed at the edge centres of the primal grid box and point tangential to this edge.

The generalized Gauss theorem is invoked for evaluating the vorticity components on the grid

$$
\int_{V} \nabla \times \mathbf{v d} \tau=-\oint_{S} \mathbf{v} \times \mathrm{d} \mathbf{s}
$$

The RHS of this equation is discretely represented by a sum over the faces of a dual grid box. Contravariant vectors with covariant measure numbers are chosen if discretised in a terrain-following coordinate system (e.g. Lagally and Franz, 1964, provide further details of integral theorems and transformation rules between coordinate systems). The divergence operator is similarly defined via the Gauss theorem

$$
\int_{V} \nabla \cdot \mathbf{g d} \tau=\oint_{S} \mathbf{g} \cdot \mathrm{d} \mathbf{s}
$$

where $\mathbf{g}$ is an arbitrary flux. The discretized version ends up in a sum over the faces of a primal grid box for the RHS that uses contravariant base vectors for the surface elements and covariant ones for the fluxes. Bonaventura and Ringler, 2005, provide details on the use of integral theorems for the discretisation in the ICON model.

\subsection{Spatial discretisation of the brackets}

For the discretisation of the Poisson and Nambu brackets, the divergence and the rotation operators are the only ones needed. In particular, the gradient operator follows as the dual counterpart of the divergence operator. This duality is connected with the foundation on which the two previously introduced Poisson brackets are built, namely the rule of integration by parts. That rule is also the background of the Arakawa Jacobian (cf. Salmon and Talley, 1989; Salmon, 2007). The discretised form of the mass bracket reads

$$
\begin{aligned}
& \{\mathcal{F}, \mathcal{H}\}_{\varrho} \Rightarrow \\
& -\sum_{m=1}^{M}\left[\left.\frac{\delta \mathcal{F}}{\delta \varrho}\right|_{m}\left(\nabla \cdot \frac{\delta \mathcal{H}}{\delta \mathbf{v}}\right)_{m}-\left.\frac{\delta \mathcal{H}}{\delta \varrho}\right|_{m}\left(\nabla \cdot \frac{\delta \mathcal{F}}{\delta \mathbf{v}}\right)_{m}\right] V_{m} \\
& =-\sum_{m=1}^{M}\left[\left.\frac{\delta \mathcal{F}}{\delta \varrho}\right|_{m}\left(\nabla \cdot \frac{\delta \mathcal{H}}{\delta \mathbf{v}}\right)_{m}+\left(\frac{\delta \mathcal{F}}{\delta \mathbf{v}} \cdot \nabla \frac{\delta \mathcal{H}}{\delta \varrho}\right)_{m}\right] V_{m},
\end{aligned}
$$

where the boundary conditions as in the analytical case are tacitly taken into account. Consequently, the divergence and the gradient operators obey a discrete version of

$$
\int_{V} \psi \nabla \cdot \mathbf{g d} \tau=-\int_{V} \mathbf{g} \cdot \nabla \psi \mathrm{d} \tau
$$

for arbitrary vector fluxes $\mathbf{g}$ and scalars $\psi$. The consequence for the model designer is that the divergence operator already defines the gradient operator. There is no direct reference to the gradient operator. It will be created automatically when writing down the equations for the prognostic variables for which $\delta \mathcal{F} / \delta \mathbf{v}$ does not vanish.

We have assumed so far that the functional derivatives $\delta \mathcal{H} / \delta \varrho$ are located in the centre $m$ of the primal grid box and that those of $\delta \mathcal{H} / \delta \mathbf{v}$ are defined as normal vector components at the faces $n$. But we have not yet defined the Hamiltonian on the grid, so that our assumptions are initially speculative. For the evaluation of the Hamiltonian, especially its kinetic energy part, and also the helicity functional, we need the definition of an inner product at the centre point of the primal grid. This is obtained by inspecting the role of the inner product in $\nabla \cdot \nabla \psi=\Delta \psi$. Illustratively and with direct reference to Figure 1, we restrict ourselves again to a plane and find

$$
\begin{aligned}
\left.\nabla \cdot \nabla \psi\right|_{m} & =\frac{1}{A_{m}} \sum_{n \in \mathcal{E}(m)} l_{n} \frac{\left(\psi_{o}-\psi_{m}\right)_{n}}{d_{n}} \\
& =\frac{1}{A_{m}} \sum_{n \in \mathcal{E}(m)} \frac{1}{d_{n} / 2} \frac{\left(\psi_{o}-\psi_{m}\right)_{n}}{d_{n}} \frac{l_{n} d_{n}}{2}
\end{aligned}
$$

where $A_{m}$ is the area of the primal grid surrounded by the edges $\mathcal{E}(m), l_{n}$ and $d_{n}$ are the length of the primal edges and the dual edges, respectively. The $\psi_{o}$ values refer to the outer (neighbouring) $\psi$ values. Thus, the sought-for inner product on a plane is

$$
\left.\mathbf{A} \cdot \mathbf{B}\right|_{m}=\frac{1}{A_{m}} \sum_{n \in \mathcal{E}(m)} a_{n} b_{n} \frac{A_{e, n}}{2},
$$


where $A_{e, n}=d_{n} l_{n}$ is refered to as the elemental area (the grey area in Figure 1), and $a_{n}$ and $b_{n}$ are the nomal components of the vectors at the edges. Similar considerations hold for three-dimensional volume boxes $V_{m}$ and elemental volumes $V_{e, n}$, where one could easily include terrain-following coordinates.

For obtaining the functional derivatives on the grid, we need to specify the global integrals on the grid. We first consider the helicity functional $h_{\mathrm{a}}$. For that, a spatial reconstruction of the vorticity components (originally tangential to the edges of the box) and normal vector components (originally normal to the face of the box) to a common point is required. There are only two places in the grid, for which such a reconstruction takes all contributors equally into account: for a quadrilateral grid these are the centre point of the box $m$ and the corner point $c$ of the grid box, so that we have

$$
h_{\mathrm{a}} \Rightarrow \frac{1}{2}\left(\frac{1}{2} \sum_{m=1}^{M} \overline{\mathbf{v}}^{m} \cdot{\overline{\boldsymbol{\omega}_{a}}}^{m} V_{m}+\frac{1}{2} \sum_{c=1}^{C} \overline{\mathbf{v}}^{c} \cdot{\overline{\omega_{a}}}^{c} V_{c}\right) .
$$

Strictly speaking, the discrete analogue should also satisfy the integration rule by parts as implied in the continuous case in (53), that is

$$
\nabla \cdot(\delta \mathbf{v} \times \mathbf{v})=\mathbf{v} \cdot(\nabla \times \delta \mathbf{v})-\delta \mathbf{v} \cdot(\nabla \times \mathbf{v}) .
$$

We were able to prove this from (73) for a quadrilateral grid, in which the rule $\sum a \bar{b}=\sum b \bar{a}$ may be applied in all coordinate directions. For other grids, where the coordinate lines are not linearly independent, more care has to be taken, but that is beyond the scope of the present article, where we confine the problem here to the quadrilateral mesh. In the same way, the vortex charge functional may be written

$$
\mathcal{P}_{a} \Rightarrow \frac{1}{2} \sum_{m=1}^{M}{\overline{\nabla \theta_{v}}}^{m} \cdot{\overline{\omega_{a}}}^{m} V_{m}+\frac{1}{2} \sum_{c=1}^{C}{\overline{\nabla \theta_{v}}}^{c} \cdot{\overline{\omega_{a}}}^{c} V_{c} .
$$

A similar procedure is necessary for the Hamiltonian (40). The functional derivative $\delta \mathcal{H} / \delta \mathbf{v}$ then yields directly the mass flux suitable for our divergence operator, only if the density is averaged onto each of the velocity points $n$ separately. Thus we have

$$
\mathcal{H} \Rightarrow \sum_{m=1}^{M}\left(\frac{1}{2} \bar{\varrho}^{n} \mathbf{v} \cdot \mathbf{v}\right)_{m} V_{m}+\varrho_{m}\left(\Phi+c_{v} T_{v}\right)_{m} V_{m}
$$

The discrete functionals of $\mathcal{M}$ and $\Theta_{v}$, (51), follow without difficulty and are given here for the sake of completeness:

$$
\mathcal{M} \Rightarrow \sum_{m=1}^{M} \varrho_{m} V_{m} \quad \text { and } \quad \Theta_{v} \Rightarrow \sum_{m=1}^{M} \widetilde{\theta}_{v, m} V_{m} .
$$

The spatial averaging needed in the functionals is up to the designer of the code. In general, arithmetic averaging is thought to be sufficient. But especially the reconstruction of the normal vortex vector from the tangential vortex vector leads later to quite a lot of averaging operations, which might smooth the flow field too strongly. Thus, higher-order reconstruction algorithms are worth considering in that case.

The Nambu brackets should remain antisymmetric during the discretisation process. Salmon (2005) proposed a strategy to obtain antisymmetry by weighting each cyclic permutation of the bracket arguments by one third, which recovers the Arakawa Jacobian (Arakawa, 1966) in the case of the barotropic vorticity equation. Inspection of the Nambu bracket valid for non-hydrostatic compressible dynamics reveals that no such weighting is necessary to retain the antisymmetry in the discretized form of the bracket, as will now be shown.

The helicity bracket $\left\{\mathcal{F}, h_{\mathrm{a}}, \mathcal{H}\right\},(54)$, invokes a scalar triple product. The scalar triple product can only be evaluated if its arguments are positioned at the same grid point. Again we refer here to the midpoint and upper corner point of the quadrilateral box. Then, the helicity bracket obtains the following numerical form for a quadrilateral mesh:

$$
\begin{aligned}
& \left\{\mathcal{F}, h_{\mathrm{a}}, \mathcal{H}\right\} \Rightarrow-\frac{1}{2} \sum_{(i, j, k)} \frac{V_{(i, j, k)}}{\varrho_{(i, j, k)}} \\
& \times\left[\overline { \frac { \delta \mathcal { F } } { \delta u } } _ { ( i + \frac { 1 } { 2 } , j , k ) } ^ { x } \left(\overline{\bar{\varrho}}^{z} w_{\left(i, j, k+\frac{1}{2}\right)} \bar{\omega}_{y\left(i+\frac{1}{2}, j, k+\frac{1}{2}\right)}, z\right.\right. \\
& \left.-\overline{\bar{Q}}^{y} v_{\left(i, j+\frac{1}{2}, k\right)}{ }^{y} \overline{\omega\left(i+\frac{1}{2}, j+\frac{1}{2}, k\right)} x, y\right) \\
& \left.+\overline{\frac{\delta \mathcal{F}}{\delta v}}_{\left(i, j+\frac{1}{2}, k\right)}(\ldots)+\overline{\frac{\delta \mathcal{F}}{\delta w}}_{\left(i, j, k+\frac{1}{2}\right)} z\right] \\
& -\frac{1}{2} \sum_{\left(i+\frac{1}{2}, j+\frac{1}{2}, k+\frac{1}{2}\right)} \frac{V_{\left(i+\frac{1}{2}, j+\frac{1}{2}, k+\frac{1}{2}\right)}}{\overline{\varrho_{(i, j, k)}} x, y, z} \\
& \times\left[\overline { \frac { \delta \mathcal { F } } { \delta u } } _ { ( i + 1 / 2 , j , k ) } { } ^ { y , z } \left({\overline{\bar{Q}^{z} w_{(i, j, k+1 / 2)}}}^{x, y}{\overline{\omega_{y\left(i+1 / 2, j, k+\frac{1}{2}\right.}}}^{y}\right.\right. \\
& \left.-\overline{\bar{Q}}^{y} v_{\left(i, j+\frac{1}{2}, k\right)} x, z \overline{\omega_{z\left(i+\frac{1}{2}, j+\frac{1}{2}, k\right)}} z\right) \\
& \left.+\overline{\frac{\delta \mathcal{F}}{\delta v}}_{\left(i, j+\frac{1}{2}, k\right)} x, z \quad(\ldots)+\overline{\frac{\delta \mathcal{F}}{\delta w}}_{\left(i, j, k+\frac{1}{2}\right)}{ }^{x, y} \quad(\ldots)\right] .
\end{aligned}
$$

This expression is automatically antisymmetric, because the scalar triple product of three vectors at the same grid positions is evaluated.

The discretisation of the mass bracket $\{\mathcal{F}, \mathcal{H}\}_{\varrho}$ was already introduced in (68). But now we are sure that the functional derivatives of the Hamiltonian give the values at the desired grid positions. When intending to use upstream-biased or flux-limited operators for mass divergence, caution is required. Within the bracket framework, 
the mass flux $\varrho \mathbf{v}$ is an indivisible quantity originated from the functional derivative $\delta \mathcal{H} / \delta \mathbf{v}$. Modifying the mass flux would also modify the sum forming the discrete Hamiltonian (75), and consequently, the specific kinetic energy as the functional derivative $\delta \mathcal{H} / \delta \varrho$, and, as a result, its gradient. Physical argument reveals that upstream-biased or flux-limited operators tend to be diffusive and tend to smooth out gradients involved. But Poisson brackets do not permit energy production/loss by definition. Therefore, a modified kinetic energy gradient serves as a correction to drive the system back to consistency.

A numerical treatment of the theta bracket reads

$$
\begin{array}{r}
\{\mathcal{F}, \mathcal{H}\}_{\widetilde{\theta}_{v}} \Rightarrow-\sum_{m=1}^{M}\left(\left.\frac{\delta \mathcal{F}}{\delta \widetilde{\theta}_{v}}\right|_{m}\left[\nabla \cdot\left(\theta \frac{\delta \mathcal{H}}{\delta \mathbf{v}}\right)\right]_{m}\right. \\
\left.-\left.\frac{\delta \mathcal{H}}{\delta \widetilde{\theta}_{v}}\right|_{m}\left[\nabla \cdot\left(\theta_{v} \frac{\delta \mathcal{F}}{\delta \mathbf{v}}\right)\right]_{m}\right) V_{m} .
\end{array}
$$

Here, $\theta_{v}$ is a thermodynamic quantity, but it is always needed at the velocity grid points. The kind of averaging is not dictated by the discretised brackets, but may be chosen by the model designer. In that way, we are free to use higher-order and even upstream-biased schemes for the virtual potential temperature, as long as the mass flux is determined by $\delta \mathcal{H} / \delta \mathbf{v}$. Diffusive operations coming along with flux limiters are allowed in the $\widetilde{\theta}_{v}$ equation itself, because they may not produce or destroy entropy - at least not in the limit of an ideal fluid. The only responsibility for processes changing the entropy is left to the 'physical brackets' $(\mathcal{F}, \vec{R})$ and $\left(\mathcal{F}, Q^{\left(\theta_{v}\right)}\right)$, which contain the dissipative terms and the adiabatic contributions to $\widetilde{\theta}_{v}$ related to the presence of water in the atmosphere.

\subsection{Prototype formulae}

We proceed to give prototype formulae for the different brackets in an equi-quadrilateral C/L-grid. Let us consider the helicity bracket alone and assume constant density. Then it describes the following dynamics:

$$
\begin{aligned}
& \frac{\partial u}{\partial t}=\frac{1}{2}\left({\overline{\bar{v}^{y}}}^{x}{\overline{\omega_{z}}}^{x}-{\overline{\bar{w}^{z}{\overline{\omega_{y}}}^{x, z}}}^{x}\right) \\
& +\frac{1}{2}\left({\overline{\bar{v}^{x, z}}}^{z}{\overline{\omega_{z}}}^{y, z}-{\overline{\bar{w}^{x, y} \bar{\omega}_{y}}}^{y, z}\right), \\
& \frac{\partial v}{\partial t}=\frac{1}{2}\left({\overline{\bar{w}^{z}}}^{y, z}{ }^{y}-{\overline{\bar{u}^{x}}}^{\overline{\omega_{z}}}{ }^{x}{ }^{y}\right) \\
& +\frac{1}{2}\left(\overline{\bar{w}}^{x, y}{\overline{\omega_{x}}}^{x, z}-{\overline{\bar{u}^{y, z}{\overline{\omega_{z}}}^{z}}}^{x, z}\right), \\
& \frac{\partial w}{\partial t}=\frac{1}{2}\left(\overline{\bar{u}}^{x}{\overline{\omega_{y}}}^{x, z}{ }^{z}-{\overline{\bar{v}^{y}}}_{\overline{\omega_{x}}}^{y, z}{ }^{z}\right) \\
& +\frac{1}{2}\left(\overline{\bar{u}}^{y, z}{\overline{\omega_{y}}}^{x, y}-{\overline{\bar{v}^{x, z}}}^{x}{\overline{\omega_{x}}}^{x, y}\right) .
\end{aligned}
$$

We observe here a lot of averaging. Dropping double averaging in one direction in (79) leads to a simpler scheme used by Bonaventura (2007, personal communication)

$$
\begin{aligned}
\frac{\partial u}{\partial t} & =\bar{v}^{x, y}{\overline{\omega_{z}}}^{y}-\bar{w}^{x, z}{\overline{\omega_{y}}}^{z}, \\
\frac{\partial v}{\partial t} & =\bar{w}^{y, z}{\overline{\omega_{x}}}^{z}-\bar{u}^{x, y}{\overline{\omega_{z}}}^{x} \\
\frac{\partial w}{\partial t} & =\bar{u}^{x, z}{\overline{\omega_{y}}}^{x}-\bar{v}^{y, z}{\overline{\omega_{x}}}^{y} .
\end{aligned}
$$

The difference between the two versions occurs due to accounting for three-dimensional flows in (79) rather than a formal application of a philosophy in (80) which is borrowed from the two-dimensional shallow-water regime. The lack of energy conservation in the Sadourny (1975) enstrophy conserving scheme is carried over to the three-dimensional case, whereas the version (79) conserves energy automatically. It should also be stressed that an enstrophy quantity defined similarly to the twodimensional enstrophy is not the structure-generating quantity in three-dimensional flow, where this role is taken over by the helicity. Therefore, a formal application of (79) to the two-dimensional shallow-water case would also be inadequate; it cannot conserve enstrophy.

From several studies the advantage of Sadourny's (1975) discretisation is known also in three-dimensional flow (Tripoli, 1992; Bonaventura, 2007, personal communication). Tripoli and Mayor (2000) simulate Ekman boundary-layer flow and state that the turbulence parametrization scheme is now no longer misused to get rid of numerical errors due to wrong enstrophy cascades, and so the turbulent fluxes become smaller in amount and more physically meaningful. A quantitative comparison between (79) and (80) is currently under investigation.

The other two brackets, $\{\mathcal{F}, \mathcal{H}\}_{\varrho}$ and $\{\mathcal{F}, \mathcal{H}\}_{\widetilde{\theta}_{m}}$, each appear in two different equations, the momentum equation and the continuity or some kind of pressure equation, respectively.

The gradient of the specific mechanical energy (kinetic plus potential) is explained via the mass bracket. A peculiarity is the evaluation of the specific kinetic energy from the discrete Hamiltonian (75) and the inner product used therein. In a one-dimensional C-grid approach, this requires firstly the evaluation of the specific kinetic energy at the velocity grid points and the subsequent averaging to the central points before taking the gradient, as given here as an example for an uniformly spaced grid

$$
\begin{aligned}
& -\left.\frac{\partial}{\partial x}\left(\frac{v^{2}}{2}\right)\right|_{i+\frac{1}{2}} \Rightarrow \\
& -\frac{1}{\Delta x}\left\{\frac{1}{2}\left(\frac{v_{i+\frac{3}{2}}^{2}}{2}+\frac{v_{i+\frac{1}{2}}^{2}}{2}\right)-\frac{1}{2}\left(\frac{v_{i+\frac{1}{2}}^{2}}{2}+\frac{v_{i-\frac{1}{2}}^{2}}{2}\right)\right\} .
\end{aligned}
$$

Other possibilities, such as firstly reconstructing the velocity vector at the central grid point and subsequently evaluating the specific kinetic energy, would not match 
with a consistent bracket discretisation. To complete the mass bracket discretisation, we refer again to the Hamiltonian $\mathcal{H}$ (75), where we have now to evaluate its functional derivative with respect to $\mathbf{v}$. It gives us the mass flux at the considered face of the grid box. Thus, the one-dimensional example follows as

$$
-\left.\frac{\partial}{\partial x}(\varrho v)\right|_{i} \Rightarrow-\frac{1}{\Delta x}\left(\bar{\varrho}_{i+\frac{1}{2}}^{x} v_{i+\frac{1}{2}}-\bar{\varrho}_{i-\frac{1}{2}}^{x} v_{i-\frac{1}{2}}\right) \text {. }
$$

A similar expression follows for the divergent flux of $\theta_{v}$. Since the mass flux is an indivisible quantity as the result of $\delta \mathcal{H} / \delta \mathbf{v}, \widetilde{\theta}_{v}$ cannot be used as an argument to the divergence operator, but rather $\theta_{v}$ itself as given in the following example:

$$
\begin{aligned}
& -\frac{\partial\left(\varrho v \theta_{v}\right)}{\partial x} \Rightarrow \\
& \quad-\frac{1}{\Delta x}\left(\bar{\varrho}_{i+\frac{1}{2}}^{x} v_{i+\frac{1}{2}} \bar{\theta}_{v_{i+\frac{1}{2}}}^{x}-\bar{\varrho}_{i-\frac{1}{2}}^{x} v_{i-\frac{1}{2}}{\overline{\theta_{v}}}_{i-\frac{1}{2}}^{x}\right) .
\end{aligned}
$$

The theta bracket reveals the pressure gradient term as a simple centred difference gradient of the Exner pressure together with a prefactor

$$
-\left.c_{p} \theta_{v} \nabla \pi\right|_{i+\frac{1}{2}} \Rightarrow-c_{p}{\overline{\theta_{v}}}_{i+\frac{1}{2}}^{x} \frac{\pi_{i+1}-\pi_{i}}{\Delta x}
$$

From the last two formulae, one observes that the virtual potential temperature occurs always in the same averaged manner. It becomes more obvious that a reconstructed value of $\theta_{v}$ for higher-order advection establishes the prefactor to the pressure gradient term.

After all this, the concerned reader may ask whether the averaging operations imply some spurious modes in the solution. We demonstrate here the discretisation with an Arakawa-C/Lorenz grid from which it is known to support such modes (Arakawa and Konor, 1996; Herzog and Gassmann, 2005). A closer look at the equations reveals that one crucial point is the virtual potential temperature. This variable occurs only in an averaged way and thus is exposed to spurious mode development. A possibility to cope with that issue might be to apply sophisticated reconstruction methods for $\theta_{v}$ at the face positions, or even better to introduce the vertical Charney-Phillips grid, where $\theta_{v}$ is placed at halflevels. The latter option, however, brings about possible difficulties formulating the divergence of $\theta_{v}$-flux terms.

\subsection{Temporal discretisation and conservation}

The antisymmetric brackets $\{\mathcal{F}, \mathcal{H}\}_{\varrho}$ and $\{\mathcal{F}, \mathcal{H}\}_{\widetilde{\theta}_{v}}$ each occur in two equations of the whole set, which leads to the requirement to treat them equally in a consistent time scheme. In the following, we investigate this point in more detail.

We first consider the dynamics of the mass bracket alone and omit contributions of the potential energy. The mass bracket describes the conservation of kinetic energy, and its dynamics are given by the combination of the inviscid Burgers equation and the continuity equation:

$$
\begin{aligned}
& \frac{\partial \mathbf{v}}{\partial t}=-\nabla \frac{\mathbf{v}^{2}}{2}, \\
& \frac{\partial \varrho}{\partial t}=-\nabla \cdot(\varrho \mathbf{v}) .
\end{aligned}
$$

Analytically, the conservation of kinetic energy is obtained by multiplying (84) with the mass flux. The following sequence of transformations serves us as traceback:

$$
\begin{aligned}
\varrho \mathbf{v} \cdot \frac{\partial \mathbf{v}}{\partial t}=-\varrho \mathbf{v} \cdot \nabla \frac{\mathbf{v}^{2}}{2} & =-\nabla \cdot\left(\varrho \mathbf{v} \frac{\mathbf{v}^{2}}{2}\right)+\frac{\mathbf{v}^{2}}{2} \nabla \cdot(\varrho \mathbf{v}) \\
& =-\nabla \cdot\left(\varrho \mathbf{v} \frac{\mathbf{v}^{2}}{2}\right)-\frac{\mathbf{v}^{2}}{2} \frac{\partial \varrho}{\partial t} \\
\varrho \mathbf{v} \cdot \frac{\partial \mathbf{v}}{\partial t}+\frac{\mathbf{v}^{2}}{2} \frac{\partial \varrho}{\partial t} & =\frac{\partial \varrho \mathbf{v}^{2} / 2}{\partial t}=-\nabla \cdot\left(\varrho \mathbf{v} \frac{\mathbf{v}^{2}}{2}\right) \cdot(86)
\end{aligned}
$$

The same sequence of transformations must hold in the numerical analogue. The last line in the preceding formula shows how the nonlinear conservable quantity, kinetic energy, is obtained by use of the product rule of differentiation in time. This conservation should also be obtained by discrete time integration. Considering the numerical conservation of a nonlinear quantity $A B$, we find

$$
\begin{aligned}
\frac{\partial A B}{\partial t} & \Rightarrow \frac{A^{n+1} B^{n+1}-A^{n} B^{n}}{\Delta t} \\
& =A^{n+1} \frac{B^{n+1}-B^{n}}{\Delta t}+B^{n} \frac{A^{n+1}-A^{n}}{\Delta t} .
\end{aligned}
$$

The kinetic energy in (86) is a product of three factors, and thus, the preceding formula (87) must be applied twice. The results for consistent time schemes are given in Table III.

Inspection reveals in each of the possibilities one nonlinear product of the $(n+1)$-values. Thus, the consistent time scheme would be nonlinear apart from the special case in which the mass bracket is the only contribution to the dynamics. Then, methods (A) and (C) would lead to a linear implicit solver in practice. In general practical applications, a linearisation of the time scheme is often more common. The most suitable choice in the sense of

Table III. Possibilities for a temporal discretisation of pairs of mass flux and specific kinetic energy.

\begin{tabular}{ccc}
\hline & $\mathbf{v}^{2} / 2$ & $\varrho \mathbf{v}$ \\
\hline (A) & $\mathbf{v}^{n} \cdot \mathbf{v}^{n+1} / 2$ & $\left(\varrho^{n} \mathbf{v}^{n}+\varrho^{n+1} \mathbf{v}^{n+1}\right) / 2$ \\
(B) & $\mathbf{v}^{n+1} \cdot \mathbf{v}^{n+1} / 2$ & $\left(\varrho^{n} \mathbf{v}^{n}+\varrho^{n} \mathbf{v}^{n+1}\right) / 2$ \\
(C) & $\mathbf{v}^{n} \cdot \mathbf{v}^{n} / 2$ & $\left(\varrho^{n+1} \mathbf{v}^{n}+\varrho^{n+1} \mathbf{v}^{n+1}\right) / 2$ \\
\hline
\end{tabular}


linear numerical stability and symmetry is to linearise the mass flux in method (A) to

$$
\varrho \mathbf{v} \Rightarrow\left(\varrho^{n} \mathbf{v}^{n+1}+\varrho^{n+1} \mathbf{v}^{n}\right) / 2 .
$$

The potential energy conservation also given by the mass bracket does not pose a problem to the temporal discretisation because the specific potential energy $\Phi$ is time-independent on a time-fixed grid.

Obviously, energy conversion occurs between mechanical and internal energy. In the last section we investigated the mechanical energy part represented by the mass bracket. Now we concentrate on the internal energy part, given by the theta bracket. The governing equations reduce here to the dynamics of sound waves

$$
\begin{gathered}
\frac{\partial \mathbf{v}}{\partial t}=-c_{p} \theta_{v} \nabla \pi, \\
\frac{\partial \widetilde{\theta}_{v}}{\partial t}=-\nabla \cdot\left(\varrho \mathbf{v} \theta_{v}\right) .
\end{gathered}
$$

Energy budgets may be observed by multiplying the first equation with the mass flux

$$
\begin{aligned}
\varrho \mathbf{v} \cdot \frac{\partial \mathbf{v}}{\partial t} & =-\varrho \mathbf{v} \cdot c_{p} \theta_{v} \nabla \pi \\
& =-\nabla \cdot\left(\varrho \mathbf{v} c_{p} \theta_{v} \pi\right)+c_{p} \pi \nabla \cdot\left(\varrho \mathbf{v} \theta_{v}\right) \\
& =-\nabla \cdot(\varrho \mathbf{v} h)-c_{v} \frac{\partial \tilde{\theta}_{v} \pi}{\partial t} .
\end{aligned}
$$

In this simplified and reduced case, the abbreviations $h:=c_{p} T_{v}$ and $u:=c_{v} T_{v}$ may be used. The last term in the preceding formula can be expressed in different ways

$$
c_{p} \pi \frac{\partial \widetilde{\theta}_{v}}{\partial t}=\frac{c_{v} c_{p}}{R_{\mathrm{d}}} \widetilde{\theta}_{v} \frac{\partial \pi}{\partial t}=c_{v} \frac{\partial \pi \widetilde{\theta}_{v}}{\partial t}=\frac{c_{v}}{R_{\mathrm{d}}} \frac{\partial p}{\partial t}=\frac{\partial \varrho u}{\partial t}
$$

if we write down the equations analytically. The equivalence of these expressions is not automatically given when replacing the continuous temporal derivative by a finite difference because of the nonlinear functional relationships between the different pressure variables. Ultimately, we aim at a time scheme which does not need linearisations of such nonlinear interdependencies, especially when relating the Exner pressure in the pressure gradient term to the actual prognostic pressure variable. Related approaches are susceptible to errors in the energy conservation. Therefore, we pursue a way from the only practicable and linearized form we were able to find using (87):

$$
\begin{aligned}
& c_{v} \frac{\left(\widetilde{\theta}_{v} \pi\right)^{n+1}-\left(\tilde{\theta}_{v} \pi\right)^{n}}{\Delta t} \\
& =c_{v} \pi^{n+1} \frac{\widetilde{\theta}_{v}^{n+1}-\widetilde{\theta}_{v}^{n}}{\Delta t}+c_{v} \widetilde{\theta}_{v}^{n} \frac{\pi^{n+1}-\pi^{n}}{\Delta t} \\
& =c_{v} \pi^{n+1}\left[-\nabla \cdot\left(\varrho \mathbf{v} \theta_{v}\right)\right]+c_{v} \widetilde{\theta}_{v}^{n}\left[-\frac{R_{\mathrm{d}}}{c_{v}} \frac{\pi^{n}}{\widetilde{\theta}_{v}^{n}} \nabla \cdot\left(\varrho \mathbf{v} \theta_{v}\right)\right],
\end{aligned}
$$

and, by backtracking the steps in (91), it follows for the pressure gradient term

$$
c_{p} \theta_{v} \nabla \pi \Rightarrow c_{p} \theta_{v} \nabla\left(\frac{c_{v}}{c_{p}} \pi^{n+1}+\frac{R_{\mathrm{d}}}{c_{p}} \pi^{n}\right) .
$$

This result is unexpected compared to what is known from the linear stability analysis of equations not written in a bracket form, where emerging implicit weights are more technical and equally chosen (Gassmann and Her$z o g, 2007)$. The weighting factors of $1 / 2$ are often chosen for time reversibility, but in the practice of NWP one encounters largely off-centred implicit weights with the intention of attenuating unphysical noise. With the current approach we give up the formal reversibility and the second-order temporal approximation, but we retain the inherent nonlinearity by avoiding linearisations of nonlinear relationships. It is evident from the given analysis that the nonlinearity of the dynamics determines the weighting factors. Furthermore, one can notice that the pressure gradient term determines the actual prognostic thermodynamic equations in the model, namely those for $\pi$ and $\widetilde{\theta}_{v}$ equally. Consequently, prognostic equations for virtual temperature, virtual potential temperature or pressure may not serve as model equations.

In the implementation of the numerical scheme, we can rearrange the pressure gradient term to solve only one thermodynamic equation for the variable $\pi^{*}$

$$
c_{p} \theta_{v} \nabla \pi \Rightarrow c_{p} \theta_{v} \nabla \pi^{*}
$$

$$
\begin{aligned}
\pi^{*} & =\pi^{n}-\Delta t \frac{R_{\mathrm{d}}}{c_{p}} \frac{\pi^{n}}{\widetilde{\theta}_{v}^{n}} \nabla \cdot\left(\theta_{v} \varrho \mathbf{v}\right), \\
\pi^{n+1} & =\frac{c_{p} \pi^{*}-R_{\mathrm{d}} \pi^{n}}{c_{v}} .
\end{aligned}
$$

The mass flux $\varrho \mathbf{v}$ in (95) has a temporal discretisation obtained via the constraint of the mass bracket. The time level of $\theta$ is optional and thus appropriately chosen at time level $n$.

The last topic to be considered within the consistent approach is the time scheme for the helicity bracket. In fact, the time scheme requires the nonlinear product

$$
\omega_{a} \times \mathbf{v} \Rightarrow \frac{1}{4}\left(\omega_{a}^{n+1}+\omega_{a}^{n}\right) \times\left(\mathbf{v}^{n+1}+\mathbf{v}^{n}\right)
$$

for its evaluation. This might easily be verified by applying (87) for the kinetic energy budget in a constant density flow regime for simplicity:

$$
\begin{aligned}
\frac{1}{2} \frac{\mathbf{v}^{(n+1) 2}-\mathbf{v}^{(n) 2}}{\Delta t} & =\mathbf{v}^{n+1 / 2} \cdot \frac{\mathbf{v}^{n+1}-\mathbf{v}^{n}}{\Delta t} \\
& =-\mathbf{v}^{n+1 / 2} \cdot\left(\omega_{\mathrm{a}}^{n+1 / 2} \times \mathbf{v}^{n+1 / 2}\right) .
\end{aligned}
$$

The scalar triple product can only vanish if also the time levels are equal. Similar arguments are valid when 
considering the helicity budget. Linearisation of (97) yields

$$
\omega_{\mathrm{a}} \times \mathbf{v} \Rightarrow \frac{1}{2}\left(\omega_{\mathrm{a}}^{n+1} \times \mathbf{v}^{n}+\omega_{\mathrm{a}}^{n} \times \mathbf{v}^{n+1}\right),
$$

which is also needed for numerical stability of the forward-in-time integration of the Coriolis terms and the implicit treatment of vertical advection terms. Both requirements are known from literature (e.g. Pielke, 1984). In the practice of modelling, the helicity term is the greatest challenge to the efficiency of the model. It requires not only cumbersome spatial averaging procedures but also a complicated implicit time scheme. In the shallow-water model world, it is sufficient to estimate the vorticity at an intermediate step by employing the vorticity equation (Bonaventura and Ringler, 2005), which appears as simple advection. However, in the three-dimensional world, the vector vorticity equation may no longer be efficiently used in that context, because it contains baroclinic terms not present in the shallowwater equations. Those terms would need much more computational effort. Unfortunately, there seems no way to overcome this dilemma. An explicit leapfrog time integration scheme would only be an option for a horizontal model part, but the Nambu bracket is inherently three-dimensional, and splitting off the equations into horizontal and vertical parts would destroy the structural background described by the antisymmetry.

So far, we introduced some small approximations to a consistent space and time discretisation, to linearise the time scheme in (88) and (99). Numerical experiments have to be carried out to evaluate the error of this manipulation. As a first pilot study, a one-dimensional experiment follows in section 6 , to investigate the scheme with respect to the linearisation applied.

\section{One-dimensional experiments with $\{\mathcal{F}, \mathcal{H}\}_{\varrho}$ and $\{\mathcal{F}, \mathcal{H}\}_{\tilde{\theta}_{v}}$}

The simplest experiments with Poisson bracket discretisations are one-dimensional. The mass bracket dynamics introduced in (84) and (85) serve us as a first example. Since our method is inviscid and does not produce entropy by definition, the developing shock will not be dissipated. Here, we perform an idealized experiment with symbolic (and thus dimensionless) initial conditions of the form

$$
\begin{aligned}
& v^{0}(x)=1.5+0.5 \cos (4 \pi x / L), \\
& \varrho^{0}(x)=0.5+0.1 \sin (2 \pi x / L) .
\end{aligned}
$$

The domain of length $L$ comprises 60 grid points with $\Delta x=3 ; 40$ time steps of size $\Delta t=1$ are performed, and the Courant-Friedrichs-Lewy value allows a maximum $v$ of 3 . We perfomed five runs with different model discretisations. Two versions, (A) and (C), are taken from Table III, and a third (D) uses the linearized model suggested in Equation (88). Additionally, we interchange the temporal discretisations for the mass flux and the specific kinetic energy from methods (A) and (C); version (E) uses the kinetic energy from (A) and the mass flux from $(\mathrm{C})$, version $(\mathrm{F})$ works vice versa. It is obvious that these last two realizations are not consistent, but it is interesting to study them also. Method (B) from Table III is nonlinear and is thus not considered.

The results of the integrated energy are shown in Figure 2. We observe that the consistent Nambu bracket versions (A) and (C) conserve energy throughout the integration time. The linearized version (D) exhibits only a marginal increase of energy, and the inconsistent versions (E) and (F) show no energy conservation. Version (F) even breaks down completely. This behaviour is a result of the linear instability occuring in version (F) because of the explicit integration of the kinetic energy gradient term. Linear instability occurs already in the same way in version (C). Imposed energy conservation masks this effect, but it is visible at time step 35 in Figure 3. The velocities in (C) and (F) indicate jumps over the maximal allowed velocity of 3 and exhibit high-amplitude noise which afterwards collapses. The model states (A) and (D) are very similar, though version (D) has somewhat larger density amplitudes. Comparing all panels of Figure 3 together, the version (E) seems the best, because it has only marginal fluctuations in the density, but the energetics are wrong, as known from Figure 2. The divergence operator - chosen only with poor second-order accuracy and without flux limiting - drives the density values even into the negative range. This is not an inherent shortcoming of the method. In addition to the energetics, we checked also the mass conservation in all the five runs; it is very accurate, with errors only in the order of machine accuracy.

This simple experiment using only the mass bracket has indicated that the linearised time discretisation (D) behaves reasonably well regarding the conservation properties. Thus, we can put forward the matter and add

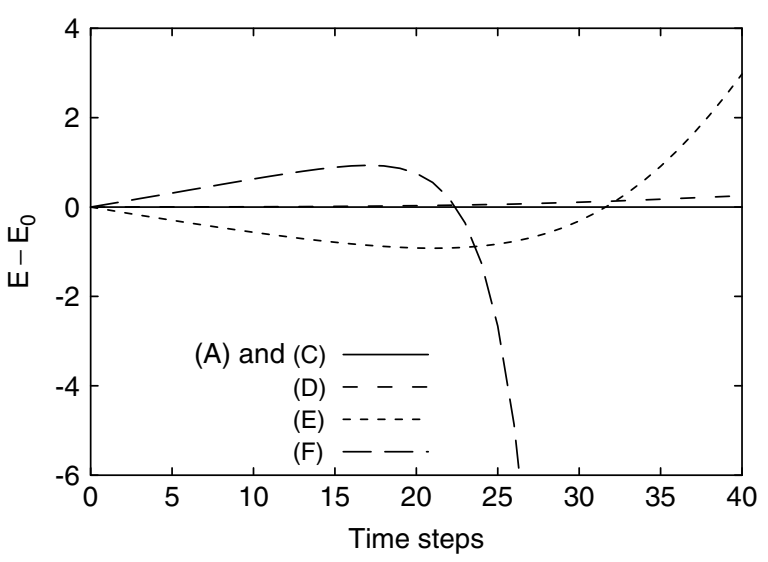

Figure 2. Integrated energy time evolution for the five mass bracket experiments (see text). 

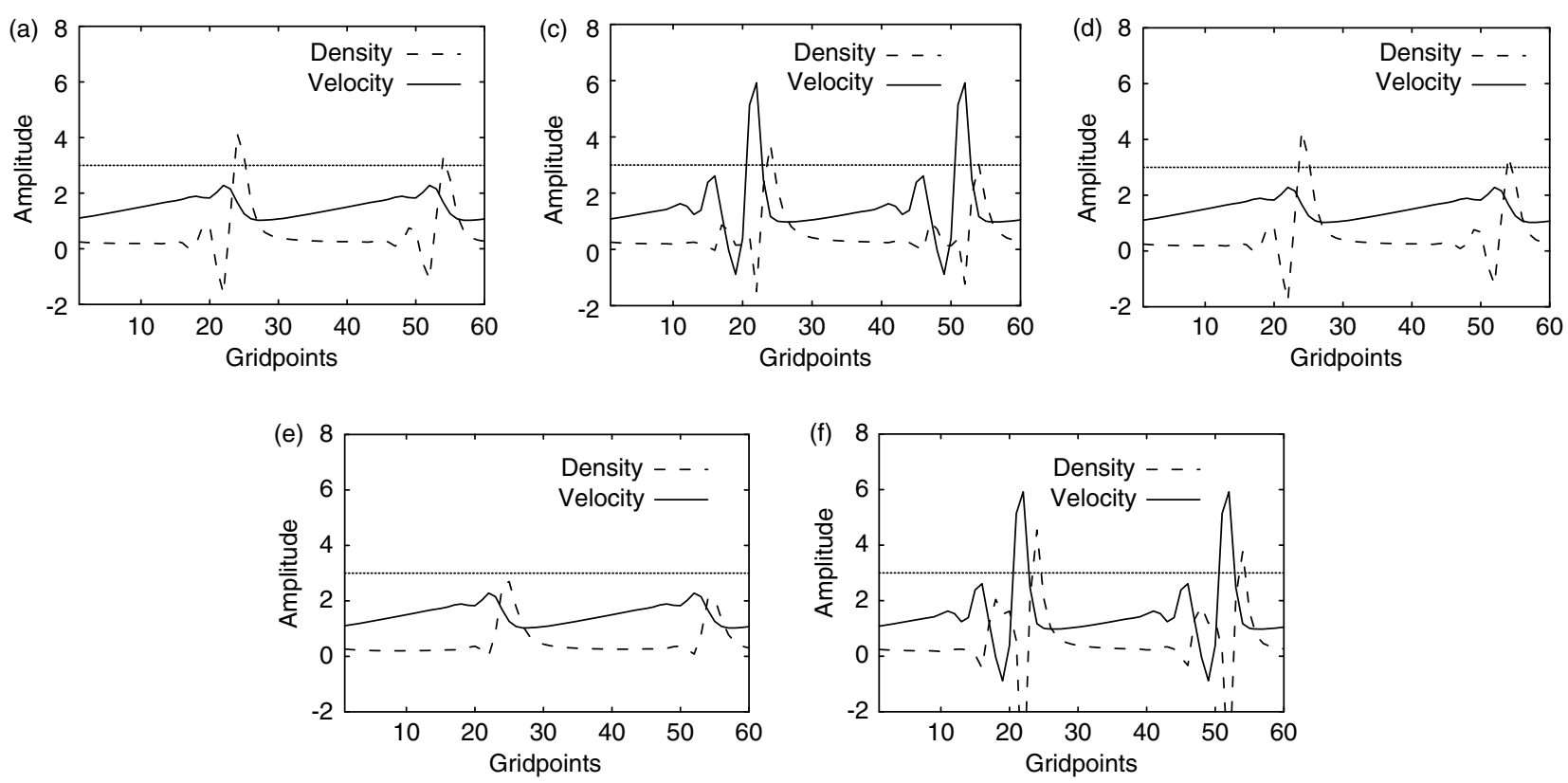

Figure 3. Model states at time step 35 for the five mass bracket experiments (see text). The critical CFL value of 3 is indicated by a thin line.

the theta bracket $\{\mathcal{F}, \mathcal{H}\}_{\tilde{\theta}}$ to our simple equation system. For comparison, we perform an experiment suggested by Satoh (2002) in which a vertically propagating sound wave is investigated. The initial state is given by an isothermal temperature profile with $T_{0}=250 \mathrm{~K}$ in hydrostatic state at rest. A pressure perturbation of $p^{\prime}=100 \mathrm{~Pa}$ is superimposed in a layer between 2.5 and $5 \mathrm{~km}$ at initial time. The numerical grid consists of 30 equally spaced vertical levels with $\Delta z=500 \mathrm{~m}$, the time step is 2 seconds. We set rigid lids at the lower and upper boundaries. Figure 4(a) shows the development of energy differences to the initial state in the vertical column during 100 seconds of integration. Total, kinetic, and available (internal plus potential) energies are plotted. An exact conversion between available and kinetic energy is actually observed in Figure 4. By expanding the total energy scale, Figure 4(b) indicates not an exact conservation, but the variations of total energy are only $10^{-4}$ times as large as the physically relevant variations, and are therefore negligible. This result is remarkable. The method demonstrated works without using total energy as a prognostic variable, and it does not need to enforce energy conservation by an iterative algorithms as outlined in Satoh (2002).

\section{Conclusions and outlook}

This work demonstrates the model construction towards a new NWP and climate model over the globe. As a prerequisite for this project, as a first step we have carried out the thorough derivation of the basic equations for such a model. Our demand is to apply the compressible non-hydrostatic equations with full physics. Therefore, the equation set is formulated in turbulence-averaged form implying the relevant turbulent fluxes, considering radiation fluxes, and describing a model atmosphere consisting of dry air and water in gaseous, liquid and solid form. The essential point of these model equations is
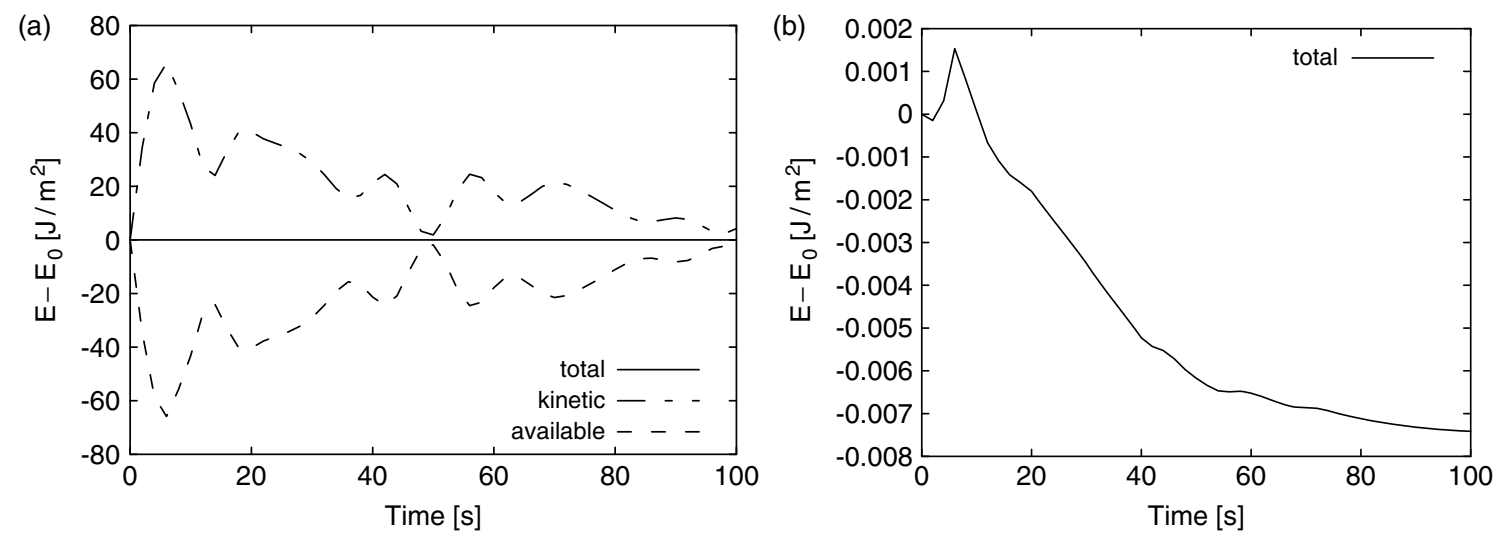

Figure 4. (a) Evolution of three kinds of energy differences in a vertical column to the initial state for a vertically propagating sound wave. (b) Evolution of the total energy difference, enlarged. 
to ensure important conservation properties concerning mass, total energy and EPV. By use of sufficient and consistent thermodynamic approximations which maintain conservation, we were able to formulate this rather general physical system in a structural form consisting of two Poisson and one Nambu brackets as an application of Névir's energy-vorticity dynamics for an ideal fluid plus further terms representing turbulent fluxes and other sources and sinks. In the given case, these bracket terms would be the only ones if the system were degraded to an ideal fluid system. It is important to point out that this formulation is valid independent of the specific parametrization process which is here deliberately left open.

Herewith we have the foundation to continue with the second step of our contribution. As known from the philosophy of Salmon (2004, 2005, 2007), Poisson and Nambu brackets offer a new way to construct the discrete analogue of a model in a systematic manner by exploiting the antisymmetric properties of these brackets in connection with conservation laws. After having provided the compressible non-hydrostatic model with full physics in an appropriate form, we adopt Salmon's basic idea to discretise the bracket part of the continuous model equations. The method for constructing the related discrete brackets is demonstrated here. It is striking that, prior to the consistent discretisation of the brackets, only very few decisions are necessary, i.e. the specification of the divergence and rotation operators and the choice of the grid type. So the principle of the discretisation process rests on maintaining the antisymmetric properties of the given brackets and the validity of the product rule of differentiation. For the shallow-water regime investigated by Salmon, and treated also by Sommer (2007, personal communication), it is sufficient to focus on the product rule of spatial differentiation, while the problem of the time scheme is not considered. In the three-dimensional case, we dealt with the time scheme included in the whole discretisation process. Thus, the product rule of time differentiation is taken into account, and it is demonstrated that the time scheme is also determined by the structure of the equations written in bracket form. Concerning the advection of scalars, the philosophy allows great freedom to introduce higher-order differencing. Total variance diminishing methods are applicable. The averaging operators in the demonstrated discretisation analysis are not kept fixed.

Many aspects of our results are probably already known or used in slightly altered form in existing models. We hope to have brought together much of this to shed new light on our conventional knowledge of modelling. We speculate that the greatest benefit from discretised brackets is expected to contribute essentially to more accurate energy cascades. Truncation errors may no longer disturb the high-wavenumber end of the spectrum, and the turbulence parametrization can act its real physical role and need not be tacitly misused as an additional computational mixing. Truncation errors may no longer lead to biases that could violate the climate of a prediction model. However, this claim together with the investigation of sufficient high local accuracy in the presented scheme is still a challenge to ensure for NWP application. There is a general consensus for climate models to use discretisations with conserving properties. We think, however, that conserving measures should not be seen as an alternative to methods with higher local accuracy, and vice versa, but both belong together. It remains to be mentioned here a philosophical problem of our approach, which implies the assumption that such a discrete representation of conservation properties meets only the ideal fluid part of the model with some possible loss of exact conservation for the full-physics system. We believe, however, that the given approach is in any case a necessary measure.

The present contribution may be seen as a general guide to build the new ICON (ICOsahedral Nonhydrostatic) global model as a joint project of the Max Planck Institute for Meteorology and the German Weather Service. We are aware of many critical details towards the goal of this project, but hope to have provided a new and interesting approach for a broadly accepted foundation.

\section{Acknowledgements}

The authors are indebted to Peter Névir (Freie Universität Berlin) and his $\mathrm{PhD}$ student Matthias Sommer for many personal discussions and contacts, from which we have learnt how to apply the Nambu theory. We thank very much Sebastian Reich (University of Potsdam) for his interest and important hints about Hamiltonian methods. We are grateful to Ulrike Wacker (AlfredWegener-Institut Bremerhaven) for her advice concerning the continuous equations of a multi-component system, and we appreciate very much Marco Giorgetta (MaxPlanck-Institut Hamburg) for his critical reading of the manuscript. Last but not least, we are grateful to three anonymous reviewers who have helped us to essentially improve the initially submitted form of this paper.

\section{References}

Arakawa A. 1966. Computational design for long-term numerical integration of the equations of fluid motion: Two-dimensional incompressible flow. Part I. J. Comput. Phys. 1: 119-143.

Arakawa A, Konor CS. 1996. Vertical differencing of the primitive equations based on the Charney-Phillips grid in hybrid $\sigma-p$ vertical coordinates. Mon. Weather Rev. 124: 511-528.

Bannon PR. 2002. Theoretical foundations for models of moist convection. J. Atmos. Sci. 59: 1967-1982.

Bannon PR. 2003. Hamiltonian description of idealized binary geophysical fluids. J. Atmos. Sci. 60: 2809-2819.

Bonaventura L, Ringler T. 2005. Analysis of discrete shallow-water models on geodesic Delauney grids with C-type staggering. Mon. Weather Rev. 133: 2351-2373.

Catry B, Geleyn J-F, Tudor M, Bénard P, Trojáková A. 2007. Flux-conservative thermodynamic equations in a mass-weighted framework. Tellus 59A(:): 71-79.

Davies T, Cullen MJP, Malcolm AJ, Mawson MH, Staniforth A, White AA, Wood N. 2005. A new dynamical core for the Met Office's global and regional modelling of the atmosphere. $Q$. J. R. Meteorol. Soc. 131: 1759-1782.

Doms G, Herbert F. 1985. Fluid- und Mikrodynamik in numerischen Modellen konvektiver Wolken. Berichte Inst. Meteorologie un Geodynamik: Univ. Frankfurt aM, Germany. 
Doms G, Schättler U. 2002. 'A description of the non-hydrostatic regional model LM. Part I: Dynamics and numerics'. Deutscher Wetterdienst: Offenbach. Available online at http://cosmo-model.cscs.ch/ public/documentation.htm\#p1.

Dubinkina S, Frank J. 2007. Statistical mechanics of Arakawa's discretizations. J. Comput. Phys. 227: 1286-1305.

Ertel H. 1942. Ein neuer hydrodynamischer Wirbelsatz. Meteorol. Z. 59: $277-281$.

Gassmann A, Herzog H-J. 2007. A consistent time-split numerical scheme applied to the non-hydrostatic compressible equations. Mon. Weather Rev. 135: 20-36.

Gyarmati I. 1970. Non-equilibrium thermodynamics. Springer: Berlin.

Herbert F. 1975. Irreversible Prozesse in der Atmosphäre, Teil 3. Contrib. Atmos. Phys. 48: 1-29.

Herzog H-J, Gassmann A. 2005. Lorenz- and Charney-Phillips vertical grid experimentation using a compressible non-hydrostatic toy model relevant to the fast-mode part of the 'Lokal-Modell'. COSMO Tech. Rep. 7, Deutscher Wetterdienst: Offenbach, Germany. Available online at http://cosmo-model.cscs.ch/public/downloads/ techReport_07.pdf.gz.

Hesselberg Th. 1925. Die Gesetze der ausgeglichenen Bewegung. Beitr. Phys. freien Atmos. 12: 141-160.

Kurgansky MV. 2006. Helicity production and maintenance in a baroclinic atmosphere. Meteorol. Z. 15: 409-416.

Lagally M, Franz W. 1964. Vorlesungen über Vektorrechnung Akad. Verlagsgesellschaft. Geest \& Portig: Leipzig.

Lange H-J. 2002. Die Physik des Wetters und des Klimas. Reimer: Berlin.

Morrison PJ. 1998. Hamiltonian description of the ideal fluid. Rev Modern Phys. 70: 467-521.

Névir P. 1998. Die Nambu-Felddarstellungen der Hydro-Thermody namik und ihre Bedeutung für die dynamische Meteorologie. Habilitationsschrift at Freie Universität Berlin, 317 pp.

Névir P. 2004. Ertel's vorticity theorems, the particle relabelling symmetry and the energy-vorticity theory of fluid mechanics. Meteorol. Z. 13: 1-14.

Névir P, Blender R. 1993. A Nambu representation of incompressible hydrodynamics using helicity and enstrophy. J. Phys. 26A(:): L1189-L1193.

Ničković S, Gavrilov MB, Tošić IA. 2002. Geostrophic adjustment on hexagonal grids. Mon. Weather Rev. 130: 668-683.
Pielke RA. 1984. Mesoscale Meteorological Modeling Academic Press. Rogers RR, Yau MK. 1989. A short course in cloud physics. Pergamon Press.

Sadourny R. 1975. The dynamics of finite difference models of the shallow-water equations. J. Atmos. Sci. 32: 680-689.

Salmon R. 2004. Poisson-bracket approach to the construction of energy- and potential-enstrophy-conserving algorithms for the shallow-water equations. J. Atmos. Sci. 61: 2016-2036.

Salmon R. 2005. A general method for conserving quantities related to potential vorticity in numerical models. Nonlinearity 18: R1-R16.

Salmon R. 2007. A general method for conserving energy and potential enstrophy in shallow-water models. J. Atmos. Sci. 64: 515-531.

Salmon R, Talley LD. 1989. Generalizations of Arakawa's Jacobian. J. Comput. Phys. 83: 247-259.

Satoh M. 2002. Conservative scheme for the compressible nonhydrostatic models with the horizontally explicit and vertically implicit time integration scheme. Mon. Weather Rev. 130: $1227-1245$

Satoh M. 2003. Conservative scheme for a compressible nonhydrostatic model with moist processes. Mon. Weather Rev. 131: 1033-1050.

Schubert WH, Hausman SA, Garcia M, Ooyama KV, Kuo H-C. 2001. Potential vorticity in a moist atmosphere. J. Atmos. Sci. 58: 3148-3157.

Thuburn J. 2008. Some conservation issues for the dynamical cores of NWP and climate models. J. Comput. Phys. 227: 3715-3730. doi:10.1016/j.jcp.2006.08.016.

Torsvik T, Thiem $\varnothing$, Berntsen J. 2005. Stability analysis of geostrophic adjustment on hexagonal grids for regions with variable depth. Mon. Weather Rev. 133: 3335-3344.

Tripoli GJ. 1992. A non-hydrostatic mesoscale model designed to simulate scale interaction. Mon. Weather Rev. 120: 1342-1359.

Tripoli GJ, Mayor SD. 2000. 'Numerical simulation of the neutral boundary layer: A comparison of enstrophy conserving with momentum conserving finite difference schemes'. Pp. 376-379 in proceedings of 14th Symposium on boundary layers and turbulence, 7-11 Aug, Aspen, CO. Available online from http://lidar.ssec.wisc.edu/papers/conferences/ekman_blt2000.pdf.

Wacker U, Frisius Th, Herbert F. 2006. Evaporation and precipitation surface effects on local mass continuity laws of moist air. J. Atmos. Sci. 63: 2642-2652. 